\title{
AREA AS THE INTEGRAL OF LENGTHS OF CONTOURS $\left({ }^{1}\right)$
}

\author{
BY \\ RAYMOND RISHEL
}

Introduction. Let $Q$ be the unit square in $E^{2}$ and $T: Q \rightarrow E^{k}$ a continuous mapping of $Q$ into Euclidean $k$-space. Let $T=1 \circ m$ be a monotone light factorization of $T$ with monotone factor $m$, light factor 1 , and middle space $M$. For $a, b \in M$ let $\Gamma(a, b, T)=\{f:[0,1] \rightarrow M: f$ is continuous and $f(0)=a$, $f(1)=b\}$. The geodesic distance, $G(a, b, T)$, between $1(\mathrm{a})$ and $1(\mathrm{~b})$ on the surface $T$ is defined by $G(a, b, T)=\inf _{f \in \Gamma(a, b, T)}$ length $1 \circ f$.

For a fixed point $p$ of $Q$, let $D(t, G, T)=\{q \in Q: G(m(p), m(q), T)>t\}$. Let $L(D(t, G, T))$ denote the length of $T$ restricted to the boundary of $D(t, G, T)$ as defined by Cesari in [2] $\left.{ }^{2}\right)$ and $A(T)$ denote the Lebesgue area of $T$. This paper investigates the relationship between $A(T)$ and $\int_{0}^{\infty} L(D(t, G, T)) d t$.

For nondegenerate mappings $T$ of finite area, Theorem III asserts that if $p$ is a point of $Q$ such that $G(m(p), b, T)$ is not infinite for every $b \in M$ different from $m(p)$ then equality holds. If $T$ is a continuous mapping of finite area and $F=\{b \in M: G(m(p), b, T)<\infty\}$ then there is a monotone retraction $r_{F}: M \rightarrow \bar{F}$ of $M$ onto the closure of $F$. If $T_{F}$ is defined by $T_{F}$ $=1 \circ r_{F} \circ m$, Theorems IV and $\mathrm{V}$ assert that $A\left(T_{F}\right)=\int_{0}^{\infty} L\left(D\left(t, G, T_{F}\right)\right)$ $=\int_{0}^{\infty} L(D(t, G, T)) d t$.

The paper consists of four parts. In Part I geodesic and $\mu_{j}$-geodesic distance are defined and the relationship between them is discussed. These concepts have been used previously by Silverman in [10] and [11]. Some properties of the length of a mapping restricted to the boundary of an open set as defined by Cesari in [2] are stated and for a connected open set an inequality between this definition and the definition given by Federer in [6] is given.

In Part II the inequality $A(T) \geqq \int_{0}^{\infty} L(D(t, G, T)) d t$ for nondegenerate mappings $T$ is proved. The use of $\mu_{j}$-geodesic distances makes it possible to give a proof of this inequality which is very similar to the proof of the Cavalieri inequality given by Cesari in [2].

Part III is concerned with proving Theorem III. Two examples are given to show the hypothesis of Theorem III are necessary. Morrey's representation theorem and a recent result found independently by Federer [7] and L. C. Young [13] are used in the proof of Theorem III.

In Part IV the cyclic additivity of $L(D(t, G, T))$ is discussed. Theorems

Received by the editors October 26, 1959.

(1) This paper consisted of the author's Ph.D. thesis at the University of Wisconsin, August 1959.

(2) The numbers in brackets refer to the bibliography at the end of the paper. 
IV and $\mathrm{V}$ are proved using Theorem III, and the cyclic additivity of Lebesgue area and $L(D(t, G, T))$.

Part I. Let $f:[0,1] \rightarrow Y$ be a continuous mapping of the unit interval into a metric space $Y$ with metric $d$. Let $t_{0} \leqq t_{1} \leqq t_{2} \leqq \cdots \leqq t_{j}$ be a collection of $j+1$ points of $[0,1]$. Let $I_{k}=\left\{t: t_{k-1} \leqq t \leqq t_{k}\right\}$ for $k=1,2,3, \cdots, j$. The following notion of length is a slight modification of a length used in [10].

Definition I. The $\mu_{j}$-length of $f$ is defined:

$$
\mu_{j} \text {-length } f=\underset{\text { as above by } j+1 \text { points }}{\sup } \sum_{k=1}^{i} \operatorname{diameter} f\left(I_{k}\right) .
$$

The following properties of $\mu_{j}$-length follow from the definition:

(a) $\mu_{j}$-length $f \leqq \mu_{j+1}$ length $f$.

(b) $\mid \mu_{j}$-length $f-\mu_{j}$-length $g \mid \leqq 2 j \sup _{t \in[0,1]} d(f(t), g(t))$.

(c) $\mu_{j}$-length $f \leqq j$ diameter $f([0,1])$.

(d) Let $f, g$ be functions from $[0,1]$ into $Y$ such that $f(1)=g(0)$.

Let $f \# g$ be defined by

$$
f \# g(t)=\left\{\begin{array}{l}
f(2 t) \text { if } 0 \leqq t \leqq \frac{1}{2} \\
g(2 t-1) \text { if } \frac{1}{2} \leqq t \leqq 1
\end{array}\right\} .
$$

Then $\mu_{j}$-length $f \# g \leqq \mu_{j}$-length $f+\mu_{j}$-length $g$.

Let $T: Q \rightarrow E^{k}$ be a continuous mapping of the unit square $Q$ in $E^{2}$ into $E^{k}$. Let $T=1 \circ m, m: Q \rightarrow M, 1: M \rightarrow E^{k}$ be a monotone light factorization of $T$ with monotone factor $m$, light factor 1 and middle space $M$. The middle space $M$ will be understood to be metrized by the metric $d(a, b)$ defined as follows: let $a$ and $b$ be points of $M$, and let $C$ denote the class of continua $K$ in $Q$ that meet $m^{-1}(a)$ and $m^{-1}(b)$, then $d(a, b)=\inf _{K \in C}$ diameter $T(K)$. For $a, b \in M$ let $\Gamma(a, b, T)=\{f:[0,1] \rightarrow M: f(0)=a, f(1)=b\}$.

Definition II. The $\mu_{j}$-geodesic distance between $T(p)$ and $T(q)$ on $T$, $\mu(j, p, q, T)$, is defined:

$$
\mu(j, p, q, T)=\inf _{f \in \Gamma(m(q), m(q), T)} \mu_{j} \text {-length } 1 \circ f .
$$

The function $\mu(j, p, q, T)$ has the following properties:

(a) $\mu(j, p, q, T) \leqq \mu(j+1, p, q, T)$.

(b) $\mu(j, p, q, T) \leqq j \inf _{f \in \Gamma(m(p), m(q), T)}$ diameter $1 \circ f([0,1])$.

(c) $\mu(j, p, \cdot, T)$ is continuous.

(d) Let $T$ and $T^{\prime}$ be continuous mappings. Let $T=1 \circ \mathrm{m}$ and $T^{\prime}$ $=1^{\prime} \circ m^{\prime}$ be monotone light factorizations of $T$ and $T^{\prime}$ with middle spaces 
$M$ and $M^{\prime}$. If there is a homeomorphism $H: M \rightarrow M^{\prime}$ such that $m^{\prime}=H \circ m$ then

$$
\left|\mu(j, p, q, T)-\mu\left(j, p, q, T^{\prime}\right)\right| \leqq 2 j\left|T-T^{\prime}\right| .
$$

(e) If $T$ is quasi linear and $q$ and $q^{\prime}$ belong to the same triangle on which $T$ is linear then

$$
\left|\mu(j, p, q, T)-\mu\left(j, p, q^{\prime}, T\right)\right| \leqq\left|T(q)-T\left(q^{\prime}\right)\right| .
$$

(f) If $T$ is Lipschitzian with Lipschitz constant $K$ then

$$
\left|\mu(j, p, q, T)-\mu\left(j, p, q^{\prime}, T\right)\right| \leqq K\left|q-q^{\prime}\right| .
$$

For each mapping $T$ two geodesic distance functions will be used, one defined on the middle space $M$, and the other defined on the parameter domain $Q$. The geodesic function on the middle space, $G(a, b, T)$, is defined by $G(a, b, T)=\inf _{f \in \Gamma(a, b, T)}$ length $1 \circ f$. The geodesic distance function defined on $Q, G(p, q, T)$, is defined by

$$
G(p, q, T)=\inf _{f \in \Gamma(m(p), m(q), T)} \text { length } 1 \circ f=G(m(p), m(q), T) .
$$

Let $\mu(p, q, T)=\lim _{j \rightarrow \infty} \mu(j, p, q, T)$. (Note that the limit exists since $\mu(j+1, p, q, T) \geqq \mu(j, p, q, T)$.)

Lemma I.1. $G(p, q, T)=\mu(p, q, T)$.

Notice that for a collection of curves it is not true that $\inf _{\alpha}$ length $f_{\alpha}$ $=\lim _{j \rightarrow \infty} \inf _{\alpha} \mu_{j}$-length $f_{\alpha}$. Let $S_{k}$ be the circle in $E^{2}$ of diameter $1 / k$ and center $(0,1 / 2 k)$. Let $f_{k}$ be the function which traverses $S_{k} k$ times starting from $(0,0)$. Then $\mu_{j}$-length $f_{k} \leqq j / k$, so inf ${ }_{k} \mu_{j}$-length $f_{k}=0$ for every $j$ but inf ${ }_{k}$ length $f_{k}=\pi$.

Proof of Lemma I.1. $G(p, q, T) \geqq \mu(j, p, q, T)$ for every $j$ hence $G(p, q, T)$ $\geqq \mu(p, q, T)$. To show the other inequality it may be assumed that $\mu(p, q, T)$ $<\infty$. Let $f_{j} \in \Gamma(m(p), m(q), T)$ be such that $\mu_{j}$-length $1 \circ f_{j}-\mu(j, p, q, T)$ $<1 / j$. Since $\mu_{k}$-length $1 \circ f_{k} \geqq \mu_{j}$-length $1 \circ f_{k}$ for $k \geqq j, \mu_{j}$-length $1 \circ f_{k}$ $<\mu(p, q, T)+1$ for $k \geqq j$.

Let $\left\{f_{\alpha}\right\}$ be a collection of functions $f_{\alpha}:[0,1] \rightarrow Y$ where $Y$ is a compact metric space. According to [8] a necessary and sufficient condition that there exist a collection $\left\{f_{\alpha}^{\prime}\right\}$ which is relatively compact in the topology of uniform convergence and such that $f_{\alpha}$ and $f_{\alpha}^{\prime}$ are Frechet equivalent is that the $\left\{f_{\alpha}\right\}$ be equally divisible i.e. for each $\epsilon>0$ there is an integer $n$ such that for each function $f_{\alpha}$ the interval $[0,1]$ can be subdivided into $n$ subintervals on which the oscillation of $f_{\alpha}$ is less than $\epsilon$. (The same $n$ must work for all the $f_{\alpha}^{\prime}$ 's.)

Let $\epsilon$ be a positive number and $N$ an integer greater than $(\mu(p, q, T)+1) \epsilon^{-1}$. For each integer $k>N$ the interval $[0,1]$ can be divided into fewer than $N$ subintervals such that oscillation $1 \circ f_{k}$ is $\leqq \epsilon$ on each interval in the following manner: 
Let $t_{1}=\sup \left\{t:\right.$ diameter $\left.1 \circ f_{k}([s: 0 \leqq s \leqq t]) \leqq \epsilon\right\}$.

Let $t_{2}=\sup \left\{t\right.$ : diameter $\left.1 \circ f_{k}\left(\left[s: t_{1} \leqq s \leqq t\right]\right) \leqq \epsilon\right\}$, etc.

There are at most $N-1$ of these $t_{i}$ 's which are less than one. Suppose not. Then

$$
\text { diameter } 1 \circ f_{k}\left(\left[t_{i} \leqq s \leqq t_{i+1}\right]\right)=\epsilon \text { if } t_{i+1}<1
$$

and

$$
\mu_{N} \text {-length } 1 \circ f_{k} \geqq N \epsilon>\mu(p, q, T)+1>\mu_{N} \text {-length } 1 \circ f_{k},
$$

a contradiction.

Since it is possible to find a number $N^{\prime}$ so that for each of the finite number of functions $1 \circ f_{1}, \cdots, 1 \circ f_{N}$ the interval $[0,1]$ may be subdivided into $N^{\prime}$ intervals on which the corresponding functions have oscillation less than $\epsilon$, it's seen that the collection $\left\{1 \circ f_{k}\right\}_{k=1}^{\infty}$ is equally divisible.

From the definition of the metric in $M$, diameter $1 \circ f_{k}\left(\left[t_{i} \leqq s \leqq t_{i+1}\right]\right)$ $\geqq$ diameter $f_{k}\left(\left[t_{i} \leqq s \leqq t_{i+1}\right]\right)$. Hence the $\left\{f_{k}\right\}$ are equally divisible. Let $\left\{f_{k}^{*}\right\}$ be a collection of functions such that $f_{k}$ and $f_{k}^{*}$ are Frechet equivalent and the collection $\left\{f_{k}^{*}\right\}$ is relatively compact. By selecting a subsequence and relabeling, it may be assumed that the $f_{k}^{*}$ converge to a function $f$. It's seen that $f_{k}^{*}$ and $f \in \Gamma(m(p), m(q), T)$ and

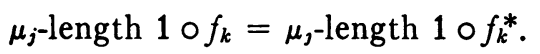

If $k \geqq j$,

$\mu_{j}$-length $1 \circ f_{k}^{*} \leqq \mu_{k}$-length $1 \circ f_{k}^{*} \leqq \mu(p, q, T)+1 / k$.

By property (b) of $\mu_{j}$-lengths

$$
\begin{gathered}
\lim _{k \rightarrow \infty} \mu_{j} \text {-length } 1 \circ f_{k}^{*}=\mu_{j} \text {-length } 1 \circ f \leqq \mu(p, q, T), \\
\lim _{j \rightarrow \infty} \mu_{j} \text {-length } 1 \circ f=\text { length } 1 \circ f \leqq \mu(p, q, T) .
\end{gathered}
$$

Hence $G(p, q, T) \leqq \mu(p, q, T)$.

Corollary I (OF THE PROOF). For $a, b, \in M$ there is an $f \in \Gamma(a, b, T)$ such that length 1 of $=\mathbf{G}(a, b, T)$.

Corollary II. $G(p, \cdot, T)$ and $G(m(p), \cdot, T)$ are lower semicontinuous.

The functions $\mu(j, p, \cdot, T)$ are an increasing sequence of continuous functions such that $\lim _{j \rightarrow \infty} \mu(j, p, q, T)=G(p, q, T)$ so $G(p, \cdot, T)$ is lower semicontinuous. $\{b \in M: G(m(p), b, T) \leqq t\}=m(\{q \in Q: G(p, q, T) \leqq t\})$ and hence is a closed set for each $t$. Therefore $G(m(p), \cdot, T)$ is lower semicontinuous.

Let $Q$ be the unit square with the relative topology as a subset of $E^{2}$. For subsets of $Q$ open, closed, boundary, etc., will be understood to be with respect to this topology. For a set $A, A^{*}$ will generally be used to denote its 
boundary. For the square $Q$ it will be necessary to speak of its boundary both using the convention mentioned above and as a subset of $E^{2}, Q^{*}$ will be used for the relative boundary and $Q^{\#}$ for the boundary as a subset of $E^{2}$.

For a continuous mapping $T: Q \rightarrow E^{k}$ and an open subset $D$ of $Q$, let $L\left(T, D^{*}\right)$ denote the length of $T$ restricted to the boundary of $D$ as defined by Cesari in [2].

The following statements about $L\left(T, D^{*}\right)$ are proved (although not stated in quite this manner) in $[1 ; 2]$ or $[3]$ or follow directly from the definition of length.

Lemma 1.2. Let $T: Q \rightarrow E^{k}$ be a continuous mapping and $D$ an open subset of $Q$.

Let $(D)_{\rho}=\{q \in Q: \operatorname{dist}(q, Q-D) \geqq \rho\}$. If $\epsilon>0$ then there is $a \rho>0$ such that if $T^{\prime}$ and $D^{\prime}$ are a continuous mapping and an open subset of $Q$ such that $\left|T-T^{\prime}\right|<\rho$ and $(D)_{\rho} \subset D^{\prime} \subset D$ then $L\left(T^{\prime}, D^{* *}\right) \geqq L\left(T, D^{*}\right)-\epsilon$.

Lemma I.3. If $D$ is an open subset of $Q$ and $T$ and $T^{\prime}$ are continuous mappings of $Q$ into $E^{k}$ such that $T\left|D^{*}=T^{\prime}\right| D^{*}$ then $L\left(T, D^{*}\right)=L\left(T^{\prime}, D^{*}\right)$.

LEMma I.4. Let $T$ and $T^{\prime}$ be Frechet equivalent mappings of $Q$ into $E^{k}$. By [9] or [5] there exist monotone light factorizations $T=1 \circ \mathrm{m}$ and $T^{\prime}=1 \circ \mathrm{m}^{\prime}$ of $T$ and $T^{\prime}$ with a common light factor 1 and a common middle space $M$. Let $D$ be an open subset of $M$ and let $D(T)$ and $D\left(T^{\prime}\right)$ be $m^{-1}(D)$ and $\left(m^{\prime}\right)^{-1}(D)$ respectively. Then $L\left(T, D(T)^{*}\right)=L\left(T^{\prime}, D\left(T^{\prime}\right)^{*}\right)$.

LEMMA I.5. Let $\alpha$ be a connected open subset of $Q$ and $T: Q \rightarrow E^{k}$ a continuous mapping. Let $\gamma$ be a component of $\alpha^{*}$ and $A(\gamma)$ be the associated open set with $\gamma$ as its boundary defined in [2]. If $L\left(T, A(\gamma)^{*}\right)<\propto$ there is a set $B$ which can be taken to be a circle or a closed interval and a function $f: B \rightarrow \gamma$ such that:

(a) $T \circ f: B \rightarrow E^{k}$ is continuous.

(b) The continua of constancy of $T$ which contain a point of $f(B)$ cover $\gamma$.

(c) $L\left(T, A(\gamma)^{*}\right)=$ length $T \circ f$.

In Part III essential use will be made of a length defined by Federer in [6]. The following lemma gives a comparison of $L\left(T, D^{*}\right)$ and this length.

Lemma I.6. Let $T: Q \rightarrow E^{k}$ be a continuous mapping. Let $\alpha$ be a connected open subset of $Q$. Let $T=1 \circ \mathrm{m}$ be a monotone light factorization of $T$ with middlespace $M$. Let $W$ denote the set of points at which $m\left(\alpha^{*}\right)$ is of positive dimension. Let $N(x, 1, W)$ equal the number, possibly infinite, of points a of $W$ such that $1(a)=x$. Let $H_{k}^{1}$ denote 1-dimensional Hausdorff measure in $E^{k}$. Then $L\left(T, \alpha^{*}\right) \geqq \int_{B^{k}} N(x, 1, W) d H_{k}^{1}$.

It is no loss of generality to assume $L\left(T, \alpha^{*}\right)<\infty$. Let $\gamma$ be a component of $\alpha^{*}$. Since $L\left(T, \alpha^{*}\right)<\infty, L\left(T, A(\gamma)^{*}\right)<\infty$ and Lemma I.5 may be applied. Let $f$ be the function described in Lemma I.5. By the theorem relating lengths and Hausdorff measure for curves 


$$
L\left(T, A(\gamma)^{*}\right)=\text { length } T \circ f=\int_{E^{k}} N(x, T \circ f, B) d H_{k}^{1} .
$$

By (b) of Lemma I.5,

$$
N(x, 1, m(\gamma))=N(x, 1, m \circ f(B)) \leqq N(x, T \circ f, B),
$$

hence

$$
L\left(T, A(\gamma)^{*}\right) \geqq \int_{E^{k}} N(x, 1, m(\gamma)) d H_{k}^{1} .
$$

Let $E=\left\{\gamma \in \alpha^{*}: L\left(T, A(\gamma)^{*}\right) \neq 0\right\}$. By 20.2(i) of [2] $L\left(T, A(\gamma)^{*}\right)=0$ if and only if $T$ is constant on $\gamma$. Since each point of $E$ belongs to a $\gamma$ on which $T$ is not constant, $m(E) \subset W$. Let $S$ be the family of all countable collections $\left\{C_{i}\right\}$ of disjoint nondegenerate continua contained in $m\left(\alpha^{*}\right)$. By Theorem 3.3 of [6]

$$
\sup _{\left\{\mathbb{C}_{1}\right\} \in S} \sum_{i=1}^{\infty} \operatorname{diameter} 1\left(C_{i}\right)=\int_{E^{k}} N(x, 1, W) d H_{k}^{1} .
$$

Let $\left\{C_{i}\right\}$ be a countable collection of disjoint nondegenerate continua of $m\left(\alpha^{*}\right)$. Since $m$ is monotone, each $C_{i}$ must be contained in some $m(\gamma)$ where $\gamma \in E$ hence

$$
\sum_{i=1}^{\infty} \operatorname{diameter} 1\left(C_{i}\right) \leqq \sum_{i=1}^{\infty} \int_{E^{k}} N\left(x, 1, C_{i}\right) d H_{k}^{1} \leqq \int_{E^{k}} N(x, 1, m(E)) d H_{k}^{1}
$$

so $\int_{E^{k}} N(x, 1, m(E)) d H_{k}^{1}=\int_{E^{k}} N(x, 1, W) d H_{k}^{1}$. Now

$$
\begin{aligned}
L\left(T, \alpha^{*}\right) & =\sum_{\gamma \ni L\left(T, A(\gamma)^{*}\right) \neq 0} L\left(T, A(\gamma)^{*}\right) \\
& \geqq \sum_{\gamma \ni L\left(T, A(\gamma)^{*}\right) \neq 0} \int_{E^{k}} N(x, 1, m(\gamma)) d H_{k}^{1} \\
& \geqq \int_{E^{k}} N(x, 1, m(E)) d H_{k}^{1}=\int_{E^{k}} N(x, 1, W) d H_{k}^{1} .
\end{aligned}
$$

Part II. Let $D\left(t, \mu_{j}, T\right)=\{q \in Q: \mu(j, p, q, T)>t\}$. Since $\mu(j, p, \cdot, T)$ is continuous $D\left(t, \mu_{j}, T\right)$ is an open set. Denote $L\left(T, D\left(t, \mu_{j}, T\right)^{*}\right)$ more briefly by $L\left(D\left(t, \mu_{j}, T\right)\right)$.

Lemma II.1. Let $T_{n}, T$ be continuous mappings such that $T_{n}$ converges uniformly to $T$ and all the $T_{n}$ 's and $T$ are light or all the $T_{n}$ 's and $T$ have $Q^{\#}$ as their only nondegenerate continuum of constancy. Then

$$
\liminf _{\tau \rightarrow t^{+}} \liminf _{n \rightarrow \infty} L\left(D\left(\tau, \mu_{j}, T_{n}\right)\right) \geqq L\left(D\left(t, \mu_{j}, T\right)\right) .
$$


Let $T_{n}=1_{n} \circ m_{n}, T=1 \circ m$ be monotone light factorizations of $T$ and $T_{n}$ with middle spaces $M$ and $M_{n}$. If $T$ and $T_{n}$ are light $m$ and $m_{n}$ are homeomorphisms. In the other case $m$ and $m_{n}$ define homeomorphisms of the quotient space $Q / Q^{\sharp}$ with $M$ and $M_{n}$. Hence property (d) of the function $\mu(j, p, q, T)$ may be used to show $\mu\left(j, p, \cdot, T_{n}\right)$ converges uniformly to $\mu(j, p, \cdot, T)$.

Let $\epsilon$ be a positive number and $\rho$ be the corresponding number for $T$, $D\left(t, \mu_{j}, T\right)$ described in Lemma I.2. Let $t^{\prime}=\min _{q \in\left(D\left(t, \mu_{j}, T\right)\right)_{p}} \mu(j, p, q, T)$. Then $t^{\prime}>t$. Let $\tau$ be any number $t<\tau<t^{\prime}$ and let $\sigma=\min \left(\tau-t, t^{\prime}-\tau\right)$. Let $N$ be an integer so that if $n>N,\left|T_{n}-T\right|<\rho$ and $\left|\mu\left(j, p, \cdot, T_{n}\right)-\mu(j, p, \cdot, T)\right|<\sigma$.

If $q \in\left(D\left(t, \mu_{j}, T\right)\right)_{\rho}$ and $n>N$

$$
\mu\left(j, p, q, T_{n}\right)>\mu(j, p, q, T)-\sigma \geqq t^{\prime}-\sigma \geqq \tau
$$

so $D\left(\tau, \mu_{j}, T_{n}\right) \supset\left(D\left(t, \mu_{j}, T\right)\right)_{\rho}$.

If $q \in D\left(\tau, \mu_{j}, T_{n}\right)$ and $n>N$

$$
\mu(j, p, q, T)>\mu\left(j, p, q, T_{n}\right)-\sigma>\tau-\sigma \geqq t
$$

so $D\left(\tau, \mu_{j}, T_{n}\right) \subset D\left(t, \mu_{j}, T\right)$.

Hence by Lemma I.2 for $n>N, L\left(D\left(\tau, \mu_{j}, T_{n}\right)\right) \geqq L\left(D\left(t, \mu_{j}, T\right)\right)-\epsilon$. Taking limits

$$
\liminf _{\tau \rightarrow t^{+}} \liminf _{n \rightarrow \infty} L\left(D\left(\tau, \mu_{j}, T_{n}\right)\right) \geqq L\left(D\left(t, \mu_{j}, T\right)\right)-\epsilon .
$$

Since $\epsilon$ was arbitrary the conclusion of the lemma follows.

Lemma II.2. Let $T: Q \rightarrow E^{k}$ be a continuous mapping. Let $D(t, G, T)$ $=\{q \in Q: G(p, q, T)>t\}$. Then $\lim \inf _{j \rightarrow \infty} L\left(D\left(t, \mu_{j}, T\right)\right) \geqq L(D(t, G, T))$.

Let $\epsilon$ be a positive number and $\rho$ be the corresponding number for $T$, $(D(t, G, T))$ described in Lemma I.2. Since $\mu(j, p, q, T) \leqq G(p, q, T), D\left(t, \mu_{j}, T\right)$ $\subset D(t, G, T)$. Since $\lim _{j \rightarrow \infty} \mu(j, p, q, T)=G(p, q, T)$ the sets $D\left(t, \mu_{j}, T\right)$ cover $D(t, G, T)$. Since $(D(t, G, T))_{\rho}$ is compact there are a finite number of sets $D\left(t, \mu_{j}, T\right)$ which cover $(D(t, G, T))_{\rho}$. Let $J$ be the largest of the integers $j$ corresponding to these sets $D\left(t, \mu_{j}, T\right)$. Since $\mu(j+1, p, q, T) \geqq \mu(j, p, q, T)$. $(D(t, G, T))_{p} \subset D\left(t, \mu_{j}, T\right)$ for $j>J$.

By Lemma I.2, $L\left(D\left(t, \mu_{j}, T\right)\right) \geqq L(D(t, G, T))-\epsilon$ for $j>J$. Hence $\lim \inf _{j \rightarrow \infty} L\left(D\left(t, \mu_{j}, T\right)\right) \geqq L(D(t, G, T))-\epsilon$ and since $\epsilon$ was arbitrary the conclusion of the lemma follows.

Lemma II.3. Let $T: Q \rightarrow E^{k}$ be a quasi linear mapping then $A(T)$ $\geqq \int_{0}^{\infty} L\left(D\left(t, \mu_{j}, T\right)\right) d t$.

Let $\Omega=\left\{\tilde{\Delta}_{i}\right\}$ be a subdivision of $Q$ into triangles on which $T$ is linear. Let $M$ be a Lipschitz constant for $T$. Let $\tilde{\Delta}_{i}$ be a triangle so that $T\left(\tilde{\Delta}_{i}\right)$ is a nondegenerate triangle in $E^{k}$. Then $T \mid \tilde{\Delta}_{i}$ has an inverse $\left(T \mid \tilde{\Delta}_{i}\right)^{-1}: T\left(\tilde{\Delta}_{i}\right) \rightarrow \tilde{\Delta}_{i}$ which is linear and hence Lipschitzian on $T\left(\tilde{\Delta}_{i}\right)$. Let $N$ be the maximum of the Lipschitz constants of the $\left(T \mid \tilde{\Delta}_{i}\right)^{-1}$. 
Let $S_{n}$ denote a strip of width $2(2 / n)^{1 / 2}$ about the edges of the triangles of $\Omega$. Let $C_{p}$ denote the square of center $q$ and sides $1 / n$ about $q$. Let $\mu^{n}(q)$ $=1 / n^{2} \int_{c_{q}} \mu(j, p, r, T) d L_{2}(r)$. The functions $\mu^{n}(q)$ are continuously differentiable, converge uniformly to $\mu(j, p, \cdot, T)$ and using property (f) of the function $\mu(j, p, q, T)$ it is seen that

$$
\left|\mu^{n}\left(q^{\prime}\right)-\mu^{n}(q)\right| \leqq M\left|q^{\prime}-q\right| .
$$

By 20.4(ii) of [2] there is a quasi linear function $\phi^{n}: Q \rightarrow E^{1}$ such that $0<\mu(j, p, q, T)-\phi^{n}(q)<\epsilon_{n}$, where $\epsilon_{n}$ is a sequence of numbers tending to 0 , and $\left|\operatorname{grad} \phi^{n}(q)-\operatorname{grad} \mu^{n}(q)\right|<1 / N n$ at each interior point of a triangle of linearity of $\phi^{n}$.

Let $\Omega^{n}=\left\{\Delta_{i}\right\}$ be a triangulation of $Q$ so that $\phi^{n}$ and $T$ are linear on each triangle of $\Omega^{n}$, each $\Delta_{i}$ is contained in a $\tilde{\Delta}_{i}$ of $\Omega$, and $\Delta_{i}$ is contained either in $S_{n}$ or $Q-S_{n}$.

Let $\Delta_{i}$ be a triangle of $\Omega^{n}$ such that $T\left(\Delta_{i}\right)$ is a nondegenerate triangle. Let $D\left(T \mid \Delta_{i}\right)^{-1}(x)$ be the differential of $\left(T \mid \Delta_{i}\right)^{-1}$ at a point $x$ of $T\left(\Delta_{i}\right)$.

$\| \operatorname{grad} \phi^{n} \circ\left(T \mid \Delta_{i}\right)^{-1}(x)|-| \operatorname{grad} \mu^{n} \circ\left(T \mid \Delta_{i}\right)^{-1}(x)||$

$=\left\|\operatorname{grad} \phi^{n}\left[D\left(T \mid \Delta_{i}\right)^{-1}(x)\right]|-| \operatorname{grad} \mu^{n}\left[D\left(T \mid \Delta_{i}\right)^{-1}(x)\right]\right\|$

$\leqq\left|\operatorname{grad} \phi^{n}\left[D\left(T \mid \Delta_{i}\right)^{-1}(x)\right]-\operatorname{grad} \mu^{n}\left[D\left(T \mid \Delta_{i}\right)^{-1}(x)\right]\right|$

$\leqq\left|\operatorname{grad} \phi^{n}\left(\left(T \mid \Delta_{i}\right)^{-1}(x)\right)-\operatorname{grad} \mu^{n}\left(\left(T \mid \Delta_{i}\right)^{-1}(x)\right)\right|\left|D\left(T \mid \Delta_{i}\right)^{-1}(x)\right|$

$$
\leqq(1 / N n) N=1 / n \text {. }
$$

Hence

$$
\left|\operatorname{grad} \phi^{n} \circ\left(T \mid \Delta_{i}\right)^{-1}(x)\right| \leqq\left|\operatorname{grad} \mu^{n} \circ\left(T \mid \Delta_{i}\right)^{-1}(x)\right|+1 / n .
$$

If $q, q^{\prime}$ belong to a triangle of $\Omega^{n}$ contained in $Q-S_{n}$ then

$$
\begin{aligned}
\left|\mu^{n}(q)-\mu^{n}\left(q^{\prime}\right)\right| & \\
& \leqq 1 / n^{2} \int_{C_{q}}\left|\mu(j, p, r, T)-\mu\left(j, p, r+q-q^{\prime}, T\right)\right| d L_{2}(r) \\
& \leqq 1 / n^{2} \int_{C_{q}} \mu\left(j, r, r+q-q^{\prime}, T\right) d L_{2}(r) \\
& =1 / n^{2} \int_{C_{q}}\left|T(r)-T\left(r+q-q^{\prime}\right)\right| d L_{2}(r) \\
& =1 / n^{2} \int_{C_{q}}\left|T\left(q-q^{\prime}\right)\right| d L_{2}(r)=\left|T(q)-T\left(q^{\prime}\right)\right| 1 / n^{2} \int_{C_{q}} d L_{2}(r) \\
& =\left|T(q)-T\left(q^{\prime}\right)\right| .
\end{aligned}
$$

Hence if $\Delta_{i}$ is a triangle of $\Omega^{n}$ contained in $Q-S_{n}$ so that $T\left(\Delta_{i}\right)$ is a nondegen. erate triangle, $\left|\operatorname{grad} \mu^{n} \circ\left(T \mid \Delta_{i}\right)^{-1}\right| \leqq 1$. This implies that 


$$
\left|\operatorname{grad} \phi^{n} \circ\left(T \mid \Delta_{i}\right)^{-1}(x)\right| \leqq 1+1 / n \text {. }
$$

If $\Delta_{i}$ is any triangle of $\Omega^{n}$ for which $T\left(\Delta_{i}\right)$ is nondegenerate and $x, y \in T\left(\Delta_{i}\right)$

$$
\begin{aligned}
\mid \mu^{n} \circ\left(T \mid \Delta_{i}\right)^{-1}(x)-\mu^{n} \circ & \left(T \mid \Delta_{i}\right)(y) \mid \\
& \leqq M\left|\left(T \mid \Delta_{i}\right)^{-1}(x)-\left(T \mid \Delta_{i}\right)^{-1}(y)\right| \leqq M N|x-y| .
\end{aligned}
$$

Hence $\left|\operatorname{grad} \mu^{n} \circ\left(T \mid \Delta_{i}\right)^{-1}(x)\right| \leqq M N$ which implies $\left|\operatorname{grad} \phi^{n} \circ\left(T \mid \Delta_{i}\right)^{-1}(x)\right|$ $\leqq M N+1 / n$.

Let $D\left(t, \phi^{n}\right)=\left\{q \in Q: \phi^{n}(q)>t\right\}$. By Lemma 20.4(i) of [2]

$$
(1+1 / n) A\left(T \mid \Delta_{i}\right) \geqq \int_{0}^{\infty} L\left(T \mid \Delta_{i},\left[D\left(t, \phi^{n}\right) \cap \Delta_{i}\right]^{*}\right) d t
$$

if $\Delta_{i}$ is a triangle contained in $Q-S_{n}$ so that $T\left(\Delta_{i}\right)$ is a nondegenerate triangle and

$$
(M N+1 / n) A\left(T \mid \Delta_{i}\right) \geqq \int_{0}^{\infty} L\left(T \mid \Delta_{i},\left[D\left(t, \phi^{n}\right) \cap \Delta_{i}\right]^{*}\right) d t
$$

if $\Delta_{i}$ is a triangle contained in $S_{n}$ so that $T\left(\Delta_{i}\right)$ is a nondegenerate.

Since

$$
\begin{gathered}
L\left(T, D\left(t, \phi^{n}\right)^{*}\right)=\sum_{\left\{\Delta_{i}: T\left(\Delta_{i}\right) \text { is nondegenerate }\right\}} L\left(T \mid \Delta_{i},\left[D\left(t, \phi^{n}\right) \cap \Delta_{i}\right]^{*}\right), \\
(1+1 / n) \sum_{\Delta_{i} \subset Q-s_{n}} A\left(T \mid \Delta_{i}\right)+(M N+1 / n) \sum_{\Delta_{i} \subset S_{n}} A\left(T \mid \Delta_{i}\right) \\
\geqq \int_{0}^{\infty} L\left(T, D\left(t, \phi^{n}\right)\right) d t .
\end{gathered}
$$

Since $\phi^{n}$ converges uniformly to $\mu(j, p, q, T)$ and $\phi^{n} \leqq \mu(j, p, q, T)$, Lemma I.2 implies $\lim \inf _{n \rightarrow \infty} L\left(T, D\left(t, \phi^{n}\right)\right) \geqq L\left(D\left(t, \mu_{j}, T\right)\right)$. By taking limits, applying Fatou's lemma, and noting that $\lim \inf _{n \rightarrow \infty} \sum_{\Delta_{i} \subset s_{n}} A\left(T \mid \Delta_{i}\right)=0$, it's concluded that $A(T) \geqq \int_{0}^{\infty} L\left(D\left(t, \mu_{j}, T\right)\right) d t$.

LEMMA II.4. Let $T$ be a light mapping or a mapping whose only nondegenerate continuum of constancy is $Q^{\# ;}$ then $A(T) \geqq \int_{0}^{\infty} L\left(D\left(t, \mu_{j}, T\right)\right) d t$.

By [9] if $T$ is light $T$ may be approximated by a sequence $T_{n}$ of light quasi linear mappings such that $A\left(T_{n}\right)$ converges to $A(T)$ or if $Q^{\#}$ is the only continuum of constancy of $T, T$ may be approximated by a sequence $T_{n}$ of quasi linear mappings with the same property such that $A\left(T_{n}\right)$ converges to $A(T)$.

Let $\phi(\tau)=\lim \inf _{n \rightarrow \infty} L\left(D\left(\tau, \mu_{j}, T_{n}\right)\right)$. By Lemma II.3 and Fatou's lemma

$$
A(T)=\lim _{n \rightarrow \infty} A\left(T_{n}\right) \geqq \liminf _{n \rightarrow \infty} \int_{0}^{\infty} L\left(D\left(\tau, \mu_{j}, T_{n}\right)\right) d \tau \geqq \int_{0}^{\infty} \phi(\tau) d \tau .
$$


Let $h=\tau-t$ and define $\phi(\tau)=0$ for $\tau<0$. Then $\int_{0}^{\infty} \phi(\tau) d \tau=\int_{0}^{\infty} \phi(t+h) d t$. By Lemma II.1 and Fatou's lemma

$$
\liminf _{h \rightarrow 0^{+}} \int_{0}^{\infty} \phi(t+h) d t \geqq \int_{0}^{\infty} \liminf _{h \rightarrow 0^{+}} \phi(t+h) d t \geqq \int_{0}^{\infty} L\left(D, T, \mu_{j}, T\right) d t .
$$

Hence

$$
A(T) \geqq \int_{0}^{\infty} L\left(D\left(t, \mu_{j}, T\right)\right) d t .
$$

Lemma II.5. Let $T$ be as in Lemma II.4. Then $A(T) \geqq \int_{0}^{\infty} L(D(t, G, T)) d t$.

By Lemma II.2, Lemma II.4, and Fatou's lemma

$$
\begin{aligned}
A(T) & \geqq \liminf _{j \rightarrow \infty} \int_{0}^{\infty} L\left(D\left(t, \mu_{j}, T\right)\right) \\
& \geqq \int_{0}^{\infty} \liminf _{j \rightarrow \infty} L\left(D\left(t, \mu_{j}, T\right)\right) d t \geqq \int_{0}^{\infty} L(D(t, G, T)) d t .
\end{aligned}
$$

THEOREM I. Let $T$ be an open or closed nondegenerate mapping, then $A(T)$ $\geqq \int_{0}^{\infty} L(D(t, G, T)) d t$.

Since $T$ is open or closed nondegenerate $T$ is Frechet equivalent to a mapping $T^{\prime}$ of the type described in Lemma II.4. Let $p^{\prime}$ be a point of $Q$ such that $m(p)=m^{\prime}\left(p^{\prime}\right)$ and let $D\left(t, G, T^{\prime}\right)=\left\{q \in Q: G\left(m^{\prime}\left(p^{\prime}\right), m^{\prime}(q), T^{\prime}\right)>t\right\}$. Then $m(D(t, G, T))=m^{\prime}\left(D\left(t, G, T^{\prime}\right)\right)$ and Lemma II.5 can be applied to give,

$$
A(T)=A\left(T^{\prime}\right) \geqq \int_{0}^{\infty} L\left(D\left(t, G, T^{\prime}\right)\right) d t=\int_{0}^{\infty} L(D(t, G, T)) d t .
$$

Part III. The following example shows that $\int_{0}^{\infty} L(D(t, G, T)) d t$ need not equal $A(T)$ if $A(T)$ is infinite. Let $(u, v)$ be coordinate variables in $Q$. Let $\phi:[0,1] \rightarrow E^{1}$ be a nowhere differentiable real valued function defined on the unit interval. Let $T: Q \rightarrow E^{2}$ be defined by $T(u, v)=(\phi(u), \phi(v))$.

$\phi$ is a light mapping, for if $\phi$ were constant on some nondegenerate connected set, the set would contain an interval on which $\phi$ was constant and $\phi$ would be differentiable on the interior of the interval. This implies $T$ is a light mapping. If $I: Q \rightarrow Q$ is the identity mapping of $Q$ onto $Q, T=T \circ I$ is a montone light factorization of $T$ with middle space $Q$.

Let $p, q$ be distinct points of $Q$ and $f \in \Gamma(p, q, T)$. Let $\left(x_{1}, x_{2}\right)$ be coordinates in $E_{2}$ and $f_{i}:[0,1] \rightarrow[0,1]$ be the component mappings of $f$. For $i=1,2$ let $\pi_{i}: E^{2} \rightarrow E^{1}$ be defined by $\pi_{i}\left(x_{1}, x_{2}\right)=x_{i} . \pi_{i}$ is Lipschitzian with Lipschitz constant 1 . Since $p$ and $q$ are distinct at least one of $f_{i}[0,1]$ covers a nondegenerate interval $[c, d]$. Now $\pi_{i} \circ T \circ f(t)=\phi\left(f_{i}(t)\right)$ and 


$$
\text { length } \begin{aligned}
T \circ f & =\int_{E^{2}} N(x, T \circ f,[0,1]) d H_{2}^{1} \\
& \geqq \int_{E^{1}} N\left(x_{i}, \pi_{i} \circ T \circ f,[0,1]\right) d H_{1}^{1} \\
& \geqq \int_{E^{1}} N\left(x_{i}, \phi,[c, d] d H_{1}^{1}=\infty\right.
\end{aligned}
$$

since if the last expression was finite $\phi$ would be of bounded variation on $[c, d]$ and hence differentiable almost everywhere on $[c, d]$.

This implies $G(p, q, T)=\infty$ if $p \neq q$ so $D(t, G, T)$ is the complement of the point $p$ for every $t>0$ and hence $L(D(t, G, T))=0$ for $t>0$. Therefore $\int_{0}^{\infty} L(D(t, G, T)) d t=0$.

There are many ways of showing $A(T)=\infty$. One is that if this were not so Theorem II proved below would be contradicted.

The following is an example of a mapping of finite area, such that there is a single point at an infinite geodesic distance from every other point. Let $C$ be the closed unit disc in $E^{2}$. Let $(\rho, \theta)$ be polar coordinates in $E^{2}$ and $(r, \omega, z)$ be cylindrical coordinates in $E^{3}$. Define $F: C \rightarrow E^{3}$ by $r=\rho, \omega=\theta, z=\rho \sin 1 / \rho$. Let $H$ be any homeomorphism of $Q$ onto $C$ and let $T=F \circ H$. Then point which maps into the center of $C$ is at an infinite geodesic distance from every other point of $Q$.

Most of the remainder of this part will be concerned with proving the following equality corresponding to the inequality of Theorem I.

THEOREM III. Let $T$ be an open or closed nondegenerate mapping of $Q$ into $E^{k}$ for which $A(T)<\infty$. Let $1 \circ m$ be a monotone light factorization of $T$ with middle space $M$. Let $p$ be a point of $Q$ such that $G(m(p), b, T)$ is finite for some $b \in M$ different from $m(p)$. Then $\int_{0}^{\infty} L(D(t, G, T)) d t=A(T)$.

If $T$ is a closed nondegenerate mapping $T$ is Frechet equivalent to a mapping $T^{\prime}$ which has $Q^{\#}$ as it's only nondegenerate continua of constancy [9]. By [9] or [5] there is a monotone mapping $m^{\prime}: Q \rightarrow M$ so that $1 \circ m^{\prime}$ is a monotone light factorization of $T^{\prime}$ with the same light factor 1 and middle space $M$ as in the monotone light factorization of $T$ and such that $m\left(Q^{\#}\right)$ and $m^{\prime}\left(Q^{\#}\right)$ are the same single point of $M$.

Let $Q^{\prime}$ be a square contained in $Q-Q^{\#}$. Then $T^{\prime} \mid Q^{\prime}$ is an open nondegenerate mapping, $m^{\prime} \mid Q^{\prime}$ is a homeomorphism and $1 \circ\left(m^{\prime} \mid Q^{\prime}\right)$ is a monotone light factorization of $T^{\prime} \mid Q^{\prime}$ with middle space $m^{\prime}\left(Q^{\prime}\right) \subset M$.

In order to handle the cases of open and closed nondegenerate mappings simultaneously let $T^{\prime}=T$ and $Q^{\prime}=Q$ if $T$ is open nondegenerate. Then if $T$ is either an open or closed nondegenerate mapping of finite area $T^{\prime} \mid Q^{\prime}$ is an open nondegenerate mapping of finite area. Morrey's theorem may be applied to obtain an almost conformal mapping $T^{\prime \prime}: Q \rightarrow E^{k}$ such that $T^{\prime \prime}$ and $T^{\prime} \mid Q^{\prime}$ 
are Frechet equivalent. There will be a monotone mapping $m^{\prime \prime}: Q \rightarrow m^{\prime}\left(Q^{\prime}\right)$ $C M$ such that $1 \circ \mathrm{m}^{\prime \prime}$ is a monotone light factorization of $T^{\prime \prime}$ with the same light factor as $T$ and middle space $m^{\prime}\left(Q^{\prime}\right)$ contained in the middle space $M$ of $T$.

Let $(u, v)$ be coordinate variables in $Q$ and $x_{1}, \cdots, x_{k}$ be the component mappings of $T^{\prime \prime}$. Since $T^{\prime \prime}$ is almost conformal it has the following properties: (a) $T^{\prime \prime}$ is BVT and ACT (of bounded variation and absolutely continuous in the sense of Tonelli). Hence $T^{\prime \prime}$ has partial derivatives almost everywhere in $Q$.

$$
\text { If } \begin{gathered}
E(q)=\sum_{i=1}^{k} x_{i u}^{2}=\left|\frac{\partial T^{\prime \prime}}{\partial u}\right|^{2}, \quad G(q)=\sum_{i=1}^{k} x_{i v}^{2}=\left|\frac{\partial T^{\prime \prime}}{\partial u}\right|^{2}, \\
F(q)=\sum_{i=1}^{k} x_{i u} x_{i v}=\frac{\partial T^{\prime \prime}}{\partial u} \cdot \frac{\partial T^{\prime \prime}}{\partial v},
\end{gathered}
$$

and $J(q)$ is the Jacobian matrix of the partial derivatives then $E(q)=G(q)$, and $F(q)=0$ almost everywhere in $Q$ and

$$
A\left(T^{\prime \prime}\right)=\int_{Q}|J(q)| d L_{2}=\int_{Q}\left(E(q) G(q)-F(q)^{2}\right)^{1 / 2} d L_{2}=\int_{Q} E(q) d L_{2} .
$$

Since $T^{\prime \prime}$ is ACT, for almost every parallel to the $u$-axis and for almost every parallel to the $v$-axis $T^{\prime \prime}$ restricted to this parallel is absolutely continuous as a function of one variable.

Lemma III.1. Let $d \in M, e \in m^{\prime}\left(Q^{\prime}\right)$. Let $f \in \Gamma(d, e, T)$ be such that length $1 \circ f=G(d, e, T)$. Then there is a continuum $K \subset\left(m^{\prime \prime}\right)^{-1}(f([0,1]))$ which joins $\left(m^{\prime \prime}\right)^{-1}(d)$ and $\left(m^{\prime \prime}\right)^{-1}(e)$ or $\left(m^{\prime \prime}\right)^{-1}(e)$ and $Q^{\#}$. If $T$ is open nondegenerate $K$ joins $\left(m^{\prime \prime}\right)^{-1}(d)$ and $\left(m^{\prime \prime}\right)^{-1}(e)$.

If $f[0,1] \subset m^{\prime}\left(Q^{\prime}\right)$ let $K=\left(m^{\prime \prime}\right)^{-1}(f([0,1]))$. Note that if $T$ is open nondegenerate $f([0,1])$ is always contained in $m^{\prime}\left(Q^{\prime}\right)$ since $m^{\prime}\left(Q^{\prime}\right)=M$ in this case. If $f([0,1])$ is not contained in $m^{\prime}\left(Q^{\prime}\right)$ let $\tau=\sup \left\{t: f(t) \in M-m^{\prime}\left(Q^{\prime}\right)\right\}$. Let $m^{\prime}\left(Q^{\prime}\right)^{\#}$ denote the boundary of $m^{\prime}\left(Q^{\prime}\right)$. Then $f([\tau, 1])$ meets $m\left(Q^{\prime}\right)^{\#}$. Since $m^{\prime \prime}$ is monotone and $m^{\prime}\left(Q^{\prime}\right)$ is a two cell $m^{\prime \prime}\left(Q^{\#}\right) \subset m^{\prime}\left(Q^{\prime}\right)^{\#}$ [9]. Therefore $K=\left(m^{\prime \prime}\right)^{-1} f([\tau, 1])$ joins $\left(m^{\prime \prime}\right)^{-1}(e)$ and $Q^{\#}$.

THEOREM II. Let $T: Q \rightarrow E^{k}$ be an open or closed nondegenerate mapping of finite area. Let $I=\{a \in M: G(a, b, T)=\infty$ for every $b \in M \ni b \neq a\}$. Then $G(a, b, T)<\infty$ if $a, b \in M-I$ and $(M-I)^{*}=M .{ }^{(3)}$

If $a, b$ are points of $M-I$ there are points $c, d$ of $M$ such that $a \neq c$, $b \neq d, G(a, c, T)<\infty$, and $G(b, d, T)<\infty$. If $T$ is closed nondegenerate at least one point in each of the pairs $a, c$ and $b, d$ is not equal to $m\left(Q^{\#}\right)$ say for

(3) The notation $(M-I)^{*}$ is used in place of a bar over $M-I$. 
definiteness that $a$ and $d$ are different from $m\left(Q^{\#}\right)$. Let $T^{\prime}$ and $m^{\prime}$ be the mappings described previously and let $Q^{\prime}$ be a square contained in $Q-Q^{\#}$ such that $\left(m^{\prime}\right)^{-1}(a)$ and $\left(m^{\prime}\right)^{-1}(d)$ are contained in $Q^{\prime}-Q^{\prime \#}$. If $T$ is open nondegenerate let $Q^{\prime}=Q$. Let $T^{\prime \prime}$ and $m^{\prime \prime}$ be the mappings described previously for the above square $Q^{\prime}$.

Let $K_{1}$ and $K_{2}$ be continua as described in Lemma III.1 with $d=a, e=c$ for $K_{1}$ and $d=b, e=d$ for $K_{2}$. If $T$ is closed nondegenerate $m^{\prime} \mid Q-Q^{\#}$ is a homeomorphism and hence since $m^{\prime \prime}$ is monotone $m^{\prime}\left(Q^{\prime \#}\right)=m^{\prime}\left(Q^{\prime}\right)^{\#}=m^{\prime \prime}\left(Q^{\sharp}\right)$. Then since $\left(m^{\prime}\right)^{-1}(a)$ and $\left(m^{\prime}\right)^{-1}(d)$ are contained in $Q^{\prime}-Q^{\prime \#},\left(m^{\prime \prime}\right)^{-1}(a)$ and $\left(m^{\prime \prime}\right)^{-1}(d)$ are contained in $Q-Q^{\#}$. Since $K_{1}$ joins $\left(m^{\prime \prime}\right)^{-1}(a)$ and $\left(m^{\prime \prime}\right)^{-1}(c)$ or $\left(m^{\prime \prime}\right)^{-1}(a)$ and $Q^{\#}$ and a similar statement holds for $K_{2}, K_{1}$ and $K_{2}$ are nondegenerate if $T$ is closed nondegenerate. If $T$ is open nondegenerate $K_{1}$ joins $\left(m^{\prime \prime}\right)^{-1}(a)$ and $\left(m^{\prime \prime}\right)^{-1}(c)$ and $K_{2}$ joins $\left(m^{\prime \prime}\right)^{-1}(b)$ and $\left(m^{\prime \prime}\right)^{-1}(d)$; hence $K_{1}$ and $K_{2}$ are nondegenerate in either case.

Let $S$ be the union of all parallels to the coordinate axes on which $T$ is absolutely continuous as a function of one variable. Since $S$ contains almost every parallel to each axis, $S$ separates any pair of points of $Q$. Hence $K_{1} \cap S$ and $K_{2} \cap S$ are not empty. Let $r \in K_{1} \cap S, s \in K_{2} \cap S$; then $G\left(a, m^{\prime \prime}(r), T\right)<\infty$ and $G\left(b, m^{\prime \prime}(s), T\right)<\infty$.

If $P$ is a parallel to say the $u$-axis contained in $S$ such that $T^{\prime \prime} \mid P$ is absolutely continuous then length $T^{\prime \prime}\left|P=\int_{0}^{1}\right| \partial T^{\prime \prime} / \partial u \mid d u<\infty$. Since a similar relationship holds for each parallel contained in $S, G\left(m^{\prime \prime}(r), m^{\prime \prime}(s), T\right)$ $<\infty$ for $r, s \in S$. Hence $G(a, b, T) \leqq G\left(a, m^{\prime \prime}(r), T\right)+G\left(m^{\prime \prime}(r), m^{\prime \prime}(s), T\right)$ $+G\left(m^{\prime \prime}(s), b, T\right)<\infty$ for $a, b \in M-I$.

Since $S$ is dense in $Q, m^{\prime \prime}(S)$ is dense in $m^{\prime \prime}(Q)$. If $T$ is closed nondegenerate let $\left\{Q_{n}^{\prime}\right\}$ be a sequence of squares contained in $Q-Q^{\#}$ such that $\cup_{n=1}^{\infty} Q_{n}^{\prime}$ $=Q-Q^{\#}$. If $S_{n}$ are the corresponding sets of parallels described above $\bigcup_{n=1}^{\infty} m_{n}^{\prime \prime}\left(S_{n}\right) \subset M-I$ and $\left(\bigcup_{n=1}^{\infty} m_{n}^{\prime \prime}\left(S_{n}\right)\right)^{+}=M$. If $T$ is open nondegenerate $m^{\prime \prime}(Q)=M$; hence in either case $(M-I)^{*}=M$.

It follows from Theorem II that if $T$ is an open or closed nondegenerate mapping of finite area there always is a point $p$ as described in the hypothesis of Theorem III. From now on unless stated otherwise the hypothesis of Theorem III will be'assumed and $T^{\prime}, m^{\prime}, Q^{\prime}, T^{\prime \prime}$, and $m^{\prime \prime}$ will be as defined previously.

A mapping which may be discontinuous but satisfies all the other conditions for an ACT mapping is said to be ACE (absolutely continuous in the sense of Evans).

Lemma III.2. Let $G\left(q, T^{\prime \prime}\right)=G\left(m(p), m^{\prime \prime}(q), T\right)$. Then $G\left(q, T^{\prime \prime}\right)$ is ACE.

Let $Q_{u_{0}}$ denote a subset of $S$ of the type $Q_{u_{0}}=\left\{(u, v) \in Q: u=u_{0}\right\}$. Let $v_{1} \leqq v_{2} \leqq \cdots \leqq v_{n}$ be points of $[0,1]$ and $q_{i}=\left(u_{0}, v_{i}\right)$ for $i=1,2, \cdots, n$ be the corresponding points of $Q_{u_{0}}$. Since $T^{\prime \prime}$ is absolutely continuous as a function of $v$ on $Q_{u_{0}}$ 


$$
G\left(m^{\prime \prime}\left(q_{i+1}\right), m^{\prime \prime}\left(q_{i}\right), T\right) \leqq \int_{v_{i}}^{v_{i+1}}\left|\frac{\partial T^{\prime \prime}}{\partial v}\right| d v .
$$

If $I$ is the set described in Theorem II, both $m(p)$ and $m^{\prime \prime}(S)$ are contained in $M-I$. Hence by Theorem II $G\left(m(p), m^{\prime \prime}(q), T\right)<\infty$ if $q \in S$.

$$
\begin{aligned}
& \sum_{i=1}^{n-1}\left|G\left(q_{i+1}, T^{\prime \prime}\right)-G\left(q_{i}, T^{\prime \prime}\right)\right| \\
& \quad=\sum_{i=1}^{n-1}\left|G\left(m(p), m^{\prime \prime}\left(q_{i+1}\right), T\right)-G\left(m(p), m^{\prime \prime}\left(q_{i}\right), T\right)\right| \\
& \quad \leqq \sum_{i=1}^{n-1} G\left(m^{\prime \prime}\left(q_{i+1}\right), m^{\prime \prime}\left(q_{i}\right) T\right) \leqq \sum_{i=1}^{n-1} \int_{v_{i}}^{v_{i+1}}\left|\frac{\partial T^{\prime \prime}}{\partial v}\right| d v
\end{aligned}
$$

Since similar considerations hold for analogous sets $Q_{v_{0}}$, it follows that $G\left(q, T^{\prime \prime}\right)$ is ACE.

Since $T^{\prime \prime}$ is almost conformal and $G\left(g, T^{\prime \prime}\right)$ is ACE there is a measurable set $B$ contained in $Q$ with the following properties:

(a) The partial derivatives of $T^{\prime \prime}$ and $G\left(q, T^{\prime \prime}\right)$ exist and $E(q)=G(q)$, $F(q)=0$ at every point of $B$.

(b) $B=\cup_{i+1}^{\infty} B_{i}$ where the $B_{i}$ are disjoint measurable sets on which $T^{\prime \prime}$ and $G\left(q, T^{\prime \prime}\right)$ are continuously differentiable. (A function $f: E^{m} \rightarrow E^{n}$ is said to be continuously differentiable on a set $H \subset E^{m}$ if its restriction to $H$ may be extended to $E^{m}$ so that the resulting function is continuously differentiable.)

(c) At every point of $B, T^{\prime \prime}$ and $G\left(q, T^{\prime \prime}\right)$ have regular approximate differentials, and the same set $A$ of boundaries of squares with sides parallel to the coordinate axes on which the limits are taken may be used for both functions.

(d) For each $i$ let $\phi_{i}: E^{2} \rightarrow E^{1}$ and $\psi_{i}: E^{2} \rightarrow E^{k}$ be continuously differentiable functions such that $\phi_{i}\left|B_{i}=G\left(q, T^{\prime \prime}\right)\right| B_{i}$ and $\psi_{i}\left|B_{i}=T^{\prime \prime}\right| B_{i}$. Let $D \phi_{i}(q)$ and $D \psi_{i}(q)$ denote the differentials of $\phi_{i}$ and $\psi_{i}$ at a point $q$. Let $D T^{\prime \prime}(q)$ and $D G\left(g, T^{\prime \prime}\right)$ denote the regular approximate differentials of $T^{\prime \prime}$ and $G\left(q, T^{\prime \prime}\right)$ at a point $q$. Then the linear transformation associated with the Jacobian matrix $J(q)$ of the partial derivatives of $T^{\prime \prime}, D \psi_{i}(q)$, and $D T^{\prime \prime}(q)$ are equal at every point of $B_{i}$ and the linear transformation associated with $\operatorname{grad} G\left(q, T^{\prime \prime}\right), D \phi_{i}(q)$, and $D G\left(q, T^{\prime \prime}\right)$ are equal at every point of $B_{i}$.

(e) $B$ is contained in the set $S$ of parallels to the axes on which $T^{\prime \prime}$ is absolutely continuous as a function of one variable.

(f) No point of $Q^{\#}$ is in $B$.

(g) $|J(q)| \neq 0$ at every point of $B$.

(h) $L_{2}((Q-B) \cap\{q \in Q:|J(q)| \neq 0\})=0$.

The set $B$ may be obtained as follows: Since $T^{\prime \prime}$ is almost conformal and 
$G\left(g, T^{\prime \prime}\right)$ is ACE the statement of (a) holds almost everywhere in $Q$. By Theorem I of [14] the existence of the partial derivatives of $T^{\prime \prime}$ and $G\left(g, T^{\prime \prime}\right)$ almost everywhere implies that $Q$ may be written $Q=\bigcup_{i=0}^{\infty} P_{i}$ where $L_{2}\left(P_{0}\right)$ $=0$ and the sets $P_{i}$ for $i \geqq 1$ are a disjoint sequence of closed sets such that $T^{\prime \prime} \mid P_{i}$ and $G\left(q, T^{\prime \prime}\right) \mid P_{i}$ are continuously differentiable. By 26.2 (i) and (ii) of [2] the existence of the partial derivatives of $T^{\prime \prime}$ and $G\left(q, T^{\prime \prime}\right)$ almost everywhere in $Q$ imply the statement of (c) holds at almost every point of $Q$.

Theorem 26.2 (i) of [2] implies the equality of the linear transformation associated with $J(q)$ and $D T^{\prime \prime}(q)$ and the equality of the linear transformation associated with $\operatorname{grad} G\left(g, T^{\prime \prime}\right)$ and $D G\left(q, T^{\prime \prime}\right)$ at almost every point of $Q$. It is seen that $D \phi_{i}(q)=D G\left(q, T^{\prime \prime}\right)$ and $D \psi_{i}(q)=D T^{\prime \prime}(q)$ at every point of density of $P_{i}$ where the quantities on the right of the equal sign exist, hence almost everywhere in $P_{i}$.

Hence by discarding several sets of measure 0 and the set of points where $|J(q)|=0$ from the sets $P_{i}$ for $i \geqq 1$ sets $B_{i}$ are obtained such that $B=\bigcup_{i=1}^{\infty} B_{i}$ has all the desired properties.

Lemma III.3. $\left|\operatorname{Grad} G\left(q, T^{\prime \prime}\right)\right|=E(q)^{1 / 2}$ at every point of $B$.

Consider the regular approximate differential $D T^{\prime \prime}(q)$ of $T^{\prime \prime}$ at a point $q$ of $B$. Let $w=(a, b)$ be a point of $E^{2}$. From conditions (a) and (d) it is seen that

$$
\begin{aligned}
\left|D T^{\prime \prime}(q)(w)\right| & =\left|\left(\begin{array}{cc}
x_{1 u} & x_{1 v} \\
\vdots & \vdots \\
x_{k u} & x_{k v}
\end{array}\right)^{\left(\begin{array}{l}
a \\
b
\end{array}\right)}\right|=\left(\sum_{i=1}^{k}\left(x_{i u} a+x_{i v} b\right)^{2}\right)^{1 / 2} \\
& =\left(a^{2} E(q)+b^{2} G(q)+2 a b F(q)\right)^{1 / 2}=|w| E(q)^{1 / 2} .
\end{aligned}
$$

Let $f \in \Gamma\left(m(p), m^{\prime \prime}(q), T\right)$ be such that length $1 \circ f=G\left(m(p), m^{\prime \prime}(q), T\right)$. Let $K$ be the corresponding continuum described in Lemma III.1 with $d=m(p)$ and $e=m^{\prime \prime}(q)$. Let $\left\{q_{n}\right\}$ be a sequence of points approaching $q$ so that $\left\{q_{n}\right\} \subset A \cap K$ where $A$ is the set described in property (c) of the set $B$. Since $q_{n} \in K$,

$$
\begin{aligned}
\left|G\left(q, T^{\prime \prime}\right)-G\left(q_{n}, T^{\prime \prime}\right)\right| & =\left|G\left(m(p), m^{\prime \prime}(q), T\right)-G\left(m(p), m^{\prime \prime}\left(q_{n}\right), T\right)\right| \\
& =G\left(m^{\prime \prime}(q), m^{\prime \prime}\left(q_{n}\right), T\right) .
\end{aligned}
$$

Hence

$$
\begin{aligned}
& \lim _{q_{n} \rightarrow q} \frac{\left|G\left(q, T^{\prime \prime}\right)-G\left(q_{n}, T^{\prime \prime}\right)\right|}{\left|q-q_{n}\right|} \geqq \lim _{q_{n} \rightarrow q} \frac{\left|T^{\prime \prime}\left(q_{n}\right)-T^{\prime \prime}(q)\right|}{\left|q_{n}-q\right|} \\
& \quad=\lim _{q_{n} \rightarrow q}\left|D T^{\prime \prime}(q)\left(\frac{q_{n}-q}{\left|q_{n}-q\right|}\right)\right|=E(q)^{1 / 2} .
\end{aligned}
$$


If $q=\left(u_{0}, v_{0}\right)$ let $q_{n}^{*}$ be a sequence of points approaching $q$ which belong to $Q_{u_{0}} \cap A$. Then

$$
\begin{aligned}
\left|G\left(q, T^{\prime \prime}\right)-G\left(q_{n}^{*}, T^{\prime \prime}\right)\right| & =\left|G\left(m(p), m^{\prime \prime}(q), T\right)-G\left(m(p), m^{\prime \prime}\left(q_{n}^{*}\right), T\right)\right| \\
& \leqq G\left(m^{\prime \prime}(q), m^{\prime \prime}\left(q_{n}^{*}\right), T\right) .
\end{aligned}
$$

By condition (e) of the set $B, B \subset S$. Hence

$$
\begin{aligned}
\lim _{q_{n}^{*} \rightarrow q} \frac{\left|G\left(q, T^{\prime \prime}\right)-G\left(q_{n}^{*}, T^{\prime \prime}\right)\right|}{\left|q-q_{n}^{*}\right|} & \leqq \lim _{q_{n}^{*} \rightarrow q} \frac{G\left(m^{\prime \prime}(q), m^{\prime \prime}\left(q_{n}^{*}\right), T\right)}{\left|q-q_{n}^{*}\right|} \\
& \leqq \lim _{q_{n} \rightarrow q} \frac{\left|\int_{v_{n}}^{v_{0}} E(q)^{1 / 2} d v\right|}{\left|v_{0}-v_{n}\right|}=E(q)^{1 / 2} .
\end{aligned}
$$

LEMma III.4. Let $C_{t}=\left\{q \in Q: G\left(q, T^{\prime \prime}\right)=t\right\}$. Using the preceding definitions and conventions

$$
A\left(T^{\prime \prime}\right)=\int_{0}^{\infty}\left[\int_{E^{k}} N\left(x, T^{\prime \prime}, B \cap C_{t}\right) d H_{k}^{1}\right] d t .
$$

The following theorem is a special case of a more general theorem recently found independently by Federer [7] and L. C. Young [13].

Let $F: Q \rightarrow E^{1}$ be a continuously differentiable real valued function on $Q$ and $h: Q \rightarrow E^{1}$ a nonnegative Borel measurable real valued function on $Q$. Let $A_{t}=\{q \in Q: F(q)=t\}$ and $K$ be measurable subset of $Q$. Then

$$
\int_{K} h|\operatorname{grad} F| d L_{2}=\int_{-\infty}^{\infty}\left[\int_{A_{t} \cap K} h d H_{2}^{1}\right] d t .
$$

Proof of Lemma III.4. Let $\phi_{i}$ be the function described in property (d) of the set $B$. It follows from property (d) that $B_{i} \cap\left\{q \in Q: \phi_{i}(q)=t\right\}=B_{i} \cap C_{t}$ and that $\operatorname{grad} G\left(q, T^{\prime \prime}\right)=\operatorname{grad} \phi_{i}(q)$ on $B_{i}$. Applying the above theorem with $F=\phi_{i}, h=E^{1 / 2}$, and $K=B_{i}$ it follows using Lemma III.3 that

$$
\begin{aligned}
\int_{0}^{\infty}\left[\int_{C_{t} \cap_{B_{i}}} E(q)^{1 / 2} d H_{2}^{1}\right] d t=\int_{B_{i}} E(q)^{1 / 2}\left|\operatorname{grad} \phi_{i}(q)\right| d L_{2} \\
=\int_{B_{i}} E(q)^{1 / 2}|\operatorname{grad} G(q, T)| d L_{2}=\int_{B_{i}} E(q) d L_{2}=\int_{B_{i}}|J(q)| d L_{2} .
\end{aligned}
$$

Since $|\operatorname{grad} \phi(q)|=E(q)^{1 / 2} \neq 0$ at every point of $B_{i}$ and $C_{t} \cap B_{i}$ $=B_{i} \cap\left\{q \in Q: \phi_{i}(q)=t\right\}$ the classical implicit function theorem implies that $C_{t} \cap B_{i}$ is the disjoint union of countably many sets $C_{i}$ each of which lies on a differentiable arc $\boldsymbol{\gamma}_{\boldsymbol{i}}$.

Let $u(q)$ denote a unit tangent vector to $\gamma_{i}$ at the point $q$. Let $\psi_{i}$ be the 
function described in property (d) of the set $B$ and let $D \psi_{i}(q)$ denote the differential of $\psi_{i}$ at $q$. The directional derivative of $\psi_{i}$ in the direction of the unit tangent vector $u(q)$ at the point $q$ is given by $D \psi_{i}(q)(u(q))$. By a classical theorem on Hausdorff measures

$$
\int_{C_{i}} D \psi(q)(u(q)) d H_{2}^{1}=\int_{E^{k}} N\left(x, \psi, C_{i}\right) d H_{k}^{1} .
$$

Since by property (d) of the set $B, D \psi_{i}(q)=D T^{\prime \prime}(q)$ at each point $q$ of $B_{i}$ and $T^{\prime \prime}\left|B_{i}=\psi\right| B_{i}$

$$
\int_{C_{i}} D T^{\prime \prime}(q)(u(q)) d H_{2}^{1}=\int_{E^{k}} N\left(x, T^{\prime \prime}, C_{i}\right) d H_{k}^{1} .
$$

By the argument used in Lemma III.2 $D T^{\prime \prime}(q)(u(q))=E(q)^{1 / 2}|u(q)|=E(q)^{1 / 2}$. Hence since the sets $C_{i}$ are disjoint

$$
\int_{C_{t} \cap_{B_{i}}} E(q)^{1 / 2} d H_{2}^{1}=\int_{E^{k}} N\left(x, T^{\prime \prime}, C_{t} \cap B_{i}\right) d H_{k}^{1}
$$

Hence

$$
\begin{aligned}
\int_{0}^{\infty}\left[\int_{E^{k}} N\left(x, T^{\prime \prime}, B_{i} \cap C_{t}\right) d H_{k}^{1}\right] d t & =\int_{0}^{\infty}\left[\int_{C_{t} \cap_{B_{i}}} E(q)^{1 / 2} d H_{2}^{1}\right] d t \\
& =\int_{B_{i}}|J(q)| d L_{2} .
\end{aligned}
$$

Now

$$
\begin{aligned}
A\left(T^{\prime \prime}\right) & =\int_{Q}|J(q)| d L_{2}=\int_{B}|J(q)| d L_{2} \\
& =\sum_{i=1}^{\infty} \int_{B_{i}}|J(q)| d L_{2}=\sum_{i=1}^{\infty} \int_{0}^{\infty}\left[\int_{E^{k}} N\left(x, T^{\prime \prime}, B_{i} \cap C_{t}\right) d H_{k}^{1}\right] d t \\
& =\int_{0}^{\infty}\left[\int_{E^{k}} N\left(x, T^{\prime \prime}, B \cap C_{t}\right) d H_{k}^{1}\right] d t
\end{aligned}
$$

LEMMA III.5. No point of $B$ is contained in a nondegenerate continuum of constancy of $T^{\prime \prime}$.

Suppose $q$ were a point of $B$ belonging to such a continuum $C$. Let $A$ be the set described in property (c) of the set $B$. Let $q_{n}$ be a sequence of points belonging to $C \cap A$ such that $q_{n} \neq q$ and $q_{n}$ converges to $q$. There is a point $v$ on the unit circle and subsequence $\left\{q_{n}^{\prime}\right\}$ of $q_{n}$ such that $\left(q_{n}^{\prime}-q\right) /\left|q_{n}^{\prime}-q\right|$ converges to $v$. Now 


$$
\lim _{n \rightarrow \infty}\left|\frac{T^{\prime \prime}\left(q_{n}^{\prime}\right)-T^{\prime \prime}(q)-D T^{\prime \prime}(q)\left(q_{n}^{\prime}-q\right)}{\left|q_{n}^{\prime}-q\right|}\right|=0 .
$$

Since $T^{\prime \prime}\left(q_{n}^{\prime}\right)=T^{\prime \prime}(q)$ and $D T^{\prime \prime}(q)$ is continuous,

$$
D T^{\prime \prime}(q)(v)=\lim _{n \rightarrow \infty} D T^{\prime \prime}(q)\left(\frac{q_{n}^{\prime}-q}{\left|q_{n}^{\prime}-q\right|}\right)=0
$$

contradicting $|J(q)| \neq 0$.

The following can be easily shown to hold by using the usual argument for relative maximum and minimum of functions.

Lemma III.6. Let $F: G \rightarrow E^{1}$ be a function from an open subset $G$ of $E^{n}$ into the real numbers. Let $x$ be a point of $G$ at which the partial derivatives of $F$ exist. If $F(x)=t$ and there is a neighborhood of $x$ on which $F(x) \geqq t(F(x) \leqq t)$ or there are arbitrarily small neighborhoods of $x$ on whose boundaries $F(x) \geqq t(F(x) \leqq t)$ then $\operatorname{grad} F(x)=0$.

Lemma III.7. Let $D\left(t, G, T^{\prime \prime}\right)=\left\{q \in Q: G\left(q, T^{\prime \prime}\right)>t\right\}$. Then $C_{t} \cap B$ $C D\left(t, G, T^{\prime \prime}\right)^{*}$.

If $q_{0}$ were a point of $C_{t} \cap B$ not in $D\left(t, G, T^{\prime \prime}\right)^{*}$, the interior of $Q-D\left(t, G, T^{\prime \prime}\right)$ would be a neighborhood of $q_{0}$ on which $G\left(q, T^{\prime \prime}\right) \leqq t$ and hence by Lemma III.6 grad $G\left(q_{0}, T^{\prime \prime}\right)=0$. But since $\mid$ gradient $G\left(q, T^{\prime \prime}\right) \mid$ $=E(q)^{1 / 2}$ and $E(q)=|J(q)| \neq 0$ at every point of $B$ this is a contradiction.

Lemma III.8. The point $p$ does not belong to $B$.

Since no point of $Q^{\#}$ belongs to $B$ this follows from Lemma III.6 by an argument entirely analogous to that of Lemma III.7.

The proofs of the following two lemmas are elementary.

Lemma III.9. Let $X$ be a locally connected unicoherent Hausdorff space. Let $A$ and $B$ be disjoint, nonempty, closed, connected subsets of $X$. Let $K$ be the component of $X-A$ containing $B$. Then $X-K$ is connected and hence by unicoherence $K^{*}=\bar{K} \cap[X-K]$ is a connected subset of $A^{*}$.

Lemma III.10. Let $X$ and $Y$ be compact Hausdorf spaces and $f: X \rightarrow Y$ be a continuous mapping of $X$ onto $Y$. Let $U$ be an open subset of $Y$ and $V=f^{-1}(U)$. Then $f\left(V^{*}\right)=U^{*}$.

Lemma III.11. Let $T: Q \rightarrow E^{k}$ be a continuous mapping. Let $D(t, G, T)$ $=\{a \in M: G(m(p), a, T)>t\}$. Let $\left\{\beta_{i}\right\}$ be the collection of components of $D(t, G, T)$. Then $\beta_{i}^{*}$ is a connected set. If T satisfies the hypothesis of Theorem III and $t$ is greater than $0, \beta_{\imath}^{*}$ is a nondegenerate connected set.

$M-D(t, G, T)$ is closed and connected, for if $c \in M-D(t, G, T)$ there is a function $f \in \Gamma(m(p), c, T)$ for which length $1 \circ f=G(m(p), c, T)$ and hence 
$f([0,1])$ is a connected set contained in $M-D(t, G, T)$ joining $m(p)$ and $c$. Let $e$ be a point of $\beta_{i}$. Applying Lemma III.9 with $A=M-D(t, G, T)$, $B=\{e\}$ it is seen that $\beta_{i}^{*}$ is a connected set.

If $T$ satisfies the hypothesis of Theorem III, $M$ is a 2-sphere or a 2-cell. Since $\beta_{i}$ is open $\beta_{i}^{*}$ will be a single point if and only if $M-\beta_{i}$ is the single point $m(p)$. There is a point $b \in M$ different from $m(p)$ for which $G(m(p), b, T)$ $<\infty$. Let $f \in \Gamma(m(p), b, T)$ be such that length $1 \circ f=G(m(p), b, T)$. Let $\tau=\sup \{s: G(m(p), f(s), T) \leqq t\}$. Since $t>0, f(0, \tau)$ is a subset of $M-\beta_{i}$ which is not a single point. Therefore $\beta_{i}^{*}$ is a nondegenerate set.

Lemma III.12. For $t>0$ let $D(t, G, T)=\{a \in M: G(m(p), a, T)>t\}$ and $\left\{\beta_{i}\right\}$ be the collection of components of $D(t, G, T)$. Then

$$
L(D(t, G, T)) \geqq \sum_{i=1}^{\infty} \int_{E^{k}} N\left(x, 1, \beta_{i}^{*}\right) d H_{k}^{1} .
$$

Let $\left\{\alpha_{i}\right\}$ be the collection of components of $D(t, G, T)$. Since $m$ is monotone and $D(t, G, T)=m^{-1}(D(t, G, T))$ each $\alpha_{i}$ is of the form $m^{-1}\left(\beta_{j}\right)$ for some $j$. For convenience it will be supposed that the indices have been chosen such that $\alpha_{i}=m^{-1}\left(\beta_{i}\right)$. By the additivity of the length

$$
L(D(t, G, T))=\sum_{i=1}^{\infty} L\left(T, \alpha_{i}^{*}\right) .
$$

Let $W_{i}=\left\{b \in m\left(\alpha_{i}^{*}\right)\right.$ : dimension $\left.\left(m\left(\alpha_{i}^{*}\right), b\right)>0\right\}$. By Lemma I.6 $L\left(T, \alpha_{i}^{*}\right)$ $\geqq \int_{B^{k}} N\left(x, 1, W_{i}\right) d H_{\mathrm{z}}^{1}$. By Lemma III.10 $m\left(\alpha_{i}^{*}\right)=\beta_{i}^{*}$. Since by Lemma III.11 $\beta_{i}^{*}$ is a nondegenerate connected set, $\beta_{i}^{*}=W_{i}$. Hence the conclusion of the lemma follows.

Lемма III.13. Let $t$ be a number greater than 0 . Let $\left\{\alpha_{i}^{\prime \prime}\right\}$ be the collection of components of $D\left(t, G, T^{\prime \prime}\right)$. If $L(D(t, G, T))<\infty$ then

$$
C_{t} \cap B \cap\left[D\left(t, G, T^{\prime \prime}\right)^{*}-\bigcup_{i=1}^{\infty} \alpha_{i}^{\prime \prime *}\right]
$$

is empty.

(Note that the assertion $L(D(t, G, T))<\infty$ has to do with the set $D(t, G, T)$ and the mapping $T$ while the conclusion involves the set $D\left(t, G, T^{\prime \prime}\right)$ associated with the mapping $T^{\prime \prime}$.)

Let $\left\{\gamma_{j}\right\}$ denote the collection of components of $m^{\prime}\left(Q^{\prime}\right) \cap D(t, G, T)$. Since $\left(m^{\prime \prime}\right)^{-1}\left(m^{\prime}\left(Q^{\prime}\right) \cap D(t, G, T)\right)=D\left(t, G, T^{\prime \prime}\right)$ each $\gamma_{j}$ is contained in a component $\beta_{i}$ of $D(t, G, T)$ and if $\gamma_{j}^{*}$ denotes the boundary of $\gamma_{j}$ relative to $m^{\prime}\left(Q^{\prime}\right)$ it is seen that $\gamma_{j}^{*} \subset \beta_{i}^{*}$.

By Lemma III.10 $m^{\prime \prime}\left(\alpha_{j}^{*}\right)=\gamma_{j}^{*} \subset \beta_{i}^{*}$. By Lemma III.12 


$$
\sum_{i=1}^{\infty} \int_{E^{k}} N\left(x, 1, \beta_{i}^{*}\right) d H_{k}^{1}<\infty .
$$

This implies there is an integer $j_{n}$ so that

$$
\int_{E^{k}} N\left(x, 1, \bigcup_{j-j_{n}}^{\infty} \gamma_{j}^{*}\right) d B_{k}^{1}<1 / n .
$$

Suppose $q \in C_{t} \cap B \cap\left[D\left(t, G, T^{\prime \prime}\right)^{*}-U_{i=1}^{\infty} \alpha_{i}^{\prime \prime *}\right]$. Consider the mapping $R: E^{k} \rightarrow E^{1}$ defined by $R(x)=\left|x-T^{\prime \prime}(q)\right| . R$ is Lipschitzian with Lipschitz constant 1 , hence

$$
\int_{B^{1}} N\left(y, R \circ 1, \bigcup_{j=j_{n}}^{\infty} \gamma_{j}^{*}\right) d H_{1}^{1}(y) \leqq \int_{E^{k}} N\left(x, 1, \bigcup_{j=j_{n}}^{\infty} \gamma_{j}^{*}\right) d H_{k}^{1}(x)<\frac{1}{n} .
$$

For each integer $n$ there is a number $r_{n}, 0<r_{n} \leqq 1 / n$ such that the boundary of the sphere $S\left(T^{\prime \prime}(q), r_{n}\right)$ of center $T^{\prime \prime}(q)$ and radius $r_{n}$ does not meet $1\left(\cup_{j=y_{n}}^{\infty} \gamma_{j}^{*}\right)$. If there were not $R \circ 1\left(\bigcup_{j-y_{n}}^{\infty} \gamma_{j}^{*}\right)$ would cover the interval $0<r_{n} \leqq 1 / n$ contradicting

$$
\int_{E^{1}} N\left(y, R \circ 1, \bigcup_{j=j_{n}}^{\infty} \gamma_{i}^{*}\right) d H_{1}^{1}(y)<1 / n
$$

Since $q \in B$ by Lemma III.5 $q$ does not belong to a nondegenerate continuum of constancy of $T^{\prime \prime}$. Hence the components $G_{n}$ of $\left(T^{\prime \prime}\right)^{-1}\left(S\left(T^{\prime \prime}(q), r_{n}\right)\right)$ that contain $q$ form a basis of connected neighborhoods of $q$ whose boundaries do not meet $\bigcup_{j=y_{n}}^{\infty} \alpha_{j}^{*}$. If the sets $G_{n}$ did not form a basis of neighborhoods of $q$ the intersection of their closures would be a nondegenerate continuum of constancy of $T^{\prime \prime}$ containing $q$ contradicting Lemma III.5.

Since $q \in B, q \notin Q^{\sharp}$. Since the sets $G_{n}$ are a basis of neighborhoods of $q$ it may be assumed by selecting a subsequence that $G_{n}$ is contained in $S(q, 1 / n)$ the sphere of radius $1 / n$ about $q$ and that $Q^{f}$ and $m^{\prime-1}(m(p))$ are contained in the same component of the complement of $Q-\bar{G}_{n}$. Denote this component by $K_{n}$. Let $G_{n}{ }^{\prime}=Q-\bar{K}_{n}$. By Lemma III.9 $G_{n}{ }^{\prime *}$ is connected and $G_{n}{ }^{*} \subset G_{n}^{*}$. Since $G_{n} \subset S(q, 1 / n), G_{n}{ }^{\prime} \subset S(q, 1 / n)$ and hence the sets $G_{n}{ }^{\prime}$ form a basis of neighborhoods of $q$ with connected boundaries whose boundaries do not meet $\bigcup_{j-j_{n}}^{\infty} \alpha_{j}^{\prime \prime *}$.

Let $f \in \Gamma\left(m(p), m^{\prime \prime}(q), T\right)$ be such that length $f=G\left(m(p), m^{\prime \prime}(q), T\right)$. Let $K$ be the continuum described in Lemma III.1 connecting $Q^{\#}$ and $q$ or $q$ and $\left(m^{\prime \prime}\right)^{-1}(m(p))$. Since $G\left(m(p), m^{\prime \prime}(q), T\right)=t$ and $m^{\prime \prime}(K) \subset f([0,1]), K \subset Q$ $-D\left(t, G, T^{\prime \prime}\right)$. Since $G_{n}^{\prime *}$ separates $q$ and $Q^{\#}$ or $\left(m^{\prime \prime}\right)^{-1}(m(p)), G_{n}^{\prime *} \cap K \neq 0$. Hence $G_{n}^{\prime *} \cap\left[Q-D\left(t, g, T^{\prime \prime}\right)\right] \neq 0$. This implies, since $G_{n}^{\prime *} \cap \bigcup_{j=j_{n}}^{\infty} \alpha_{j}^{\prime \prime *}=0$ and $G_{n}^{\prime *}$ is connected, that $G_{n}^{\prime *} \cap \bigcup_{j=j_{n}}^{\infty} \alpha_{j}^{\prime \prime}=0$.

Since $q \in\left[D\left(t, G, T^{\prime \prime}\right)^{*}-\bigcup_{j=1}^{\infty} \alpha^{\prime \prime *}\right], q \in Q-U_{j=1}^{j_{n}} \bar{\alpha}_{j}^{\prime \prime}$. Let 
Since

$$
H_{n}=G_{n}^{\prime} \cap\left[Q-\bigcup_{j=1}^{j_{n}} \bar{\alpha}_{j}^{\prime \prime}\right] .
$$

$$
H_{n}^{*} \subset\left[Q-U_{j=1}^{y_{n}} \bar{\alpha}_{j}^{\prime \prime}\right]^{*}, H_{n}^{*} \cap U_{j=1}^{y_{n}} \alpha_{j}^{\prime \prime}=0 .
$$

Since $H_{n}^{*} \subset G_{n}^{\prime *} \cup\left[Q-U_{j=1}^{\prime} \bar{\alpha}_{j}^{\prime \prime}\right]^{*}$ and neither of these meets $\bigcup_{j-y_{n}} \alpha_{j}^{\prime \prime}$, $H_{n}^{*} \cap U_{j=y_{n}}^{\infty} \alpha_{j}^{\prime \prime}=0$.

Thus the sets $H_{n}$ are a basis of neighborhoods of $q$ on whose boundaries $G\left(\cdot, T^{\prime \prime}\right) \leqq t$, hence by Lemma III.6 $\operatorname{grad} G\left(q, T^{\prime \prime}\right)=0$ contradicting $q \in B$.

LEMma III.14. $A\left(T^{\prime \prime}\right) \leqq \int_{0}^{\infty} L(D(t, G, T)) d t$.

By Lemma III.4 $A\left(T^{\prime \prime}\right)=\int_{0}^{\infty} \int_{B^{k}} N\left(x, T^{\prime \prime}, B \cap C_{t}\right) d H_{k}^{1} d t$. By Lemma III.12 $L(D(t, G, T)) \geqq \sum_{i=1}^{\infty} \int_{E^{k}} N\left(x, 1, \beta_{i}^{*}\right) d H_{k}^{1}$. Using Lemma III.10 it is seen that $m^{\prime \prime}\left(\alpha_{i}^{\prime \prime *}\right) \subset \beta_{j}^{*}$ for some $j$. Therefore

$$
L(D(t, G, T)) \geqq \int_{E^{k}} N\left(x, 1, m^{\prime \prime}\left(\bigcup_{j=1}^{\infty} \alpha_{j}^{\prime \prime *}\right)\right) d H_{k}^{1} .
$$

By Theorem I, $A(T) \geqq \int_{0}^{\infty} L(D(t, G, T)) d t$, hence $L(D(t, G, T))$ is finite for almost every $t$. Therefore by Lemma III.13 and Lemma III.7 $B \cap C_{t}$ $\subset U_{j=1}^{\infty} \alpha_{j}^{\prime \prime *}$ for almost every $t$. By Lemma III.5 $N\left(x, T^{\prime \prime}, B \cap C_{t}\right)$ $=N\left(x, 1, m^{\prime \prime}\left(B \cap C_{t}\right)\right)$. Hence for almost every $t$

$$
L(D(t, G, T)) \geqq \int_{E^{k}} N\left(x, 1, m^{\prime \prime}\left(\bigcup_{j=1}^{\infty} \alpha_{j}^{\prime \prime *}\right)\right) d H_{k}^{1} \geqq \int_{E^{k}} N\left(x, T^{\prime \prime}, B \cap C_{t}\right) d H_{k}^{1} .
$$

Therefore

$$
A\left(T^{\prime \prime}\right)=\int_{0}^{\infty}\left[\int_{E^{k}} N\left(x, T^{\prime \prime}, B \cap C_{t}\right) d H_{k}^{1}\right] d t \leqq \int_{0}^{\infty} L(D(t, G, T)) d t .
$$

Proof of Theorem III. If $T$ is open nondegenerate Theorem III follows from Lemma III.14 and Theorem I since $T^{\prime \prime}$ and $T$ are Frechet equivalent. If $T$ is closed nondegenerate a succession of squares $Q_{n}$ may be chosen in a manner similar to the choice of $Q^{\prime}$ so that the $Q_{n}$ expand outward to $Q$. Let $T_{n}^{\prime}$ $=T^{\prime} \mid Q_{n}$ and $T_{n}^{\prime \prime}$ be defined as $T^{\prime \prime}$ was defined. Then $A\left(T_{n}^{\prime}\right)=A\left(T_{n}^{\prime \prime}\right)$ and $A\left(T_{n}^{\prime}\right)$ converges to $A\left(T^{\prime}\right)=A(T)$. Hence using Lemma III.14

\section{Part IV.}

$$
\lim _{n \rightarrow \infty} A\left(T_{n}^{\prime \prime}\right)=A(T) \leqq \int_{0}^{\infty} L(D(t, G, T)) d t .
$$

LeMma IV.1. Let $T: Q \rightarrow E^{k}$ be a continuous mapping and $1 \circ m$ a monotone light factorization of $T$ with middle space $M$. Let $\beta$ be a component of $D(t, G, T)$. Then either $\beta^{*}$ is a single point or $\beta^{*}$ belongs to a single proper cyclic element of $M$. 
Suppose not, then there would be a pair of distinct points $a$ and $b$ of $\beta^{*}$ and a point $c$ of $M$ such that $c$ separates $a$ and $b$. By Lemma III.11 $\beta^{*}$ is connected, hence $c$ must belong to $\beta^{*}$. Since $\beta$ is open $c$ must belong to $M-\beta$. Let $A$ be the component of $M-\{c\}$ containing $a$ and $B=M-A \cup\{c\}$. Since $c$ separates $a$ and $b$ and $c \notin \beta, A \cap \beta$ and $B \cap \beta$ are nonempty sets which form a separation of $\beta$ contradicting that $\beta$ is connected.

If $C$ is a proper cyclic element of $M$ each boundary point of $C$ separates $M$ and there is a monotone retraction $r_{C}: M \rightarrow C$ of $M$ onto $C$ which takes the part of $M$ separated from the rest of $C$ by a boundary point of $C$ onto the boundary point [9]. Let $T_{C}$ be defined by $T_{C}=1 \circ r_{C} \circ \mathrm{m}$; then $1 \mid C$ and $r_{C} \circ m$ are the light and monotone factors and $C$ the middle space in a monotone light factorization of $T_{C}$.

LEMMA IV.2. Let $s_{C}=G\left(m(p), r_{C} \circ m(p), T\right)$. Let $t$ be a number greater than or equal to $s_{C}$. Let $D\left(s, G, T_{c}\right)=\left\{a \in C: G\left(r_{C} \circ m(p), a, T_{C}\right)>s\right\}$. Let $B_{C}$ be the union of the components $\beta_{i}$ of $D(t, G, T)$ such that $\beta_{i} \cap C \neq 0$. Then $B_{C}$ $=r_{C}^{-1}\left(D\left(t-s_{C}, G, T_{C}\right)\right)$.

If $m(p) \notin C, r_{C} \circ m(p)$ is a boundary point of $C$ and hence separates $m(p)$ and $C-\left\{r_{C} \circ m(p)\right\}$. If $m(p) \in C, G\left(m(p), r_{C} \circ m(p), T\right)=0$. Hence if $a \in C, G(m(p), a, T)=G\left(m(p), r_{C} \circ m(p), T\right)+G\left(r_{C} \circ m(p), a, T\right)$. Since if $a, b \in C, G(a, b, T)=G\left(a, b, T_{c}\right), G(m(p), a, T)=s_{C}+G\left(r_{C} \circ m(p), a, T_{c}\right)$ for $a \in C$.

Since each $\beta_{i}$ is connected, $\beta_{i} \cap C \neq 0$, and $r_{C}$ has the properties described above, $r_{C}\left(\beta_{i}\right) \subset \beta_{i}$. Hence if $b \in \beta_{i}$

$$
s_{C}+G\left(r_{C} \circ m(p), r_{C}(b), T_{C}\right)=G\left(m(p), r_{C}(b), T_{C}\right)>t
$$

which implies $\beta_{i} \subset r_{C}^{-1}\left(D\left(t-s_{C}, G, T_{C}\right)\right)$ for each $\beta_{i}$.

If $a \in r_{C}^{-1}\left(\boldsymbol{D}\left(t-s_{C}, \boldsymbol{G}, T_{C}\right)\right), \boldsymbol{G}\left(r_{C} \circ m(p), r_{C}(a), T\right)>0$. This implies $m(p)$ and $a$ do not belong to the same component of the complement of $C$, hence either $a \in C$ or $r_{C}(a)$ separates $m(p)$ and $a$ in $M$. Therefore $G(m(p), a, T)$ $\geqq G\left(m(p), r_{C}(a), T\right)=s_{C}+G\left(r_{C} \circ m(p), r_{C}(a), T_{C}\right)>t$. Hence $a \in D(t, G, T)$.

Since $r_{C}$ is a monotone retraction each component of $r_{C}^{-1}\left(D\left(t-s_{C}, G, T_{C}\right)\right)$ meets $C$ and hence is contained in a component $\beta_{i}$ of $B_{C}$. Therefore $B_{C}$ $=r_{C}^{-1}\left(D\left(t-s_{C}, G, T_{C}\right)\right.$.

LEMma IV.3. If $C$ is a cyclic element for which $s_{C} \leqq t$ and there is a component $\beta$ of $D(t, G, T)$ which meets $C, \beta^{*}$ meets $C$. Conversely if $\beta^{*}$ meets $C$, $s_{C} \leqq t$.

Suppose $C$ was a cyclic element for which $s_{C} \leqq t$ and a component $\beta$ of $D(t, G, T)$ met $C$ and $\beta^{*}$ did not meet $C$. Since $s_{C} \leqq t, C-\beta$ is not empty. Since $\beta^{*}$ separates $M$ and $\beta^{*} \cap C=0, \beta \cap C$ and $C-\beta$ would be a separation of $C$ contradicting that $C$ is connected.

If $b \in \beta^{*} \cap C, G(m(p), b, T) \leqq t$. If $m(p) \in C, s_{C}=G\left(m(p), r_{C} \circ m(p), T\right)=0$. 
If $m(p) \notin C, r_{C} \circ m(p)$ separates $m(p)$ and the rest of $C$ hence in either case if $\beta^{*} \cap C \neq 0, s_{C} \leqq t$.

Corollary. There is only one cyclic element $C$ for which $\beta \cap C \neq 0$ and $s_{C} \leqq t$.

By Lemma IV.1 $\beta^{*}$ is contained in a single proper cyclic element of $M$.

Lemma IV.4. Let $F=\{a \in M: G(m(p), a, T)<\infty\}$. For a proper cyclic element $C$ of $M$ let

$$
L_{C}(t)=\left\{\begin{array}{cl}
L\left(D\left(t-s_{C}, G, T_{C}\right)\right) & \text { if } s_{C} \leqq t \\
0 & \text { if } s_{C}>t .
\end{array}\right.
$$

Then $L(D(t, G, T))=\sum_{c \subset \bar{p}} L_{C}(t)$.

For each cyclic element $C$ of $M$ for which $s_{C} \leqq t$, let $B_{C}$ be the union of the components of $D(t, G, T)$ which meet $C$ and let $\{\beta\}$ be the collection of components not meeting any cyclic element $C$ for which $s_{C} \leqq t$. Let $A_{C}=m^{-1}\left(B_{C}\right)$ and $\{\alpha\}=\left\{m^{-1}(\beta)\right\}$. By Lemma IV.3 and its corollary $D(t, G, T)$ is a disjoint union of the sets of $\{\alpha\}$ and the sets $A_{c}$. By the additivity properties of the length

$$
L(D(t, G, T))=\sum_{\alpha \in\{\alpha \mid} L\left(T, \alpha^{*}\right)+\sum_{\left\{::_{\sigma^{S}} \leq t\right\}} L\left(T, A_{C^{*}}\right) .
$$

By Lemma IV.1 $\beta^{*}$ is either a single point or is contained in a proper cyclic element of $M$. From Lemma IV.3 if $\beta^{*}$ meets a proper cyclic element $C, s_{C} \leqq t$. Since $\beta \cap C=0$ for each $C$ for which $s_{C} \leqq t, \beta \subset M-C$. Then $\beta^{*}$ must be contained in $C \cap(M-C)^{*}$ which is a single point. Hence $\beta^{*}$ is always a single point of $M$. Therefore Lemma III.10 implies that $T$ is constant on $\alpha^{*}$, and hence $L\left(T, \alpha^{*}\right)=0$ for every $\alpha \in\{\alpha\}$.

By Lemma IV.2 $r_{C} \circ m^{-1}\left(D\left(t-s_{C}, G, T_{C}\right)\right)=m^{-1}\left(B_{C}\right)=A_{C}$. Since $m\left(A_{C}^{*}\right)$ $C C$, it follows from Lemma III.10 that $r_{C} \circ m\left(A_{C}^{*}\right)=m\left(A_{C}^{*}\right)=B_{C}^{*}$. Hence $T\left|A_{C}^{*}=T_{C}\right| A_{C}^{*}$ which implies by Lemma I.3 that $L\left(D\left(t-s_{C}, G, T_{C}\right)\right)$ $=L\left(T, A_{C}^{*}\right)$.

For the mapping $T_{c}$ let $I_{C}$ be the set $I$ described in Theorem II. If $C \varangle \bar{F}$ either $s_{C}$ is infinite or $r_{C} \circ m(p)$ belongs to $I_{C}$. If this were not so, by Theorem II and the fact that if $b \in C, G(m(p), b, T)=s_{C}+G\left(r_{C} \circ m(p), b, T_{C}\right), F$ would be dense in $C$ contradicting $C \Phi \bar{F}$.

If $s_{C}$ is finite then $r_{C} \circ m(p) \in I_{C}$ and hence for every $t>0, D\left(t, G, T_{C}\right)$ consists of the complement of the single point $p$. Hence $L\left(D\left(t, G, T_{c}\right)\right)=0$ for every $t$ in this case.

Combining the preceding remarks it is seen that

$$
L(D(t, G, T))=\sum_{\left\{:_{s_{C} \leq t} t \text { and } C \subset \bar{F}\right)} L\left(D\left(t-s_{C}, G, T_{C}\right)\right)=\sum_{C \subset \bar{F}} L_{C}(t) .
$$


TheOREM IV. Let $T: Q \rightarrow E^{k}$ be a continuous mapping of finite area. Let $T=1 \circ \mathrm{m}$ be a monotone light factorization of $T$ with middle space $M$. Let $C$ denote the collection of proper cyclic elements of $M$. Let $D(t, G, T), T_{c}$, and $F$ be as defined previously. Then

$$
\int_{0}^{\infty} L(D(t, G, T)) d t=\sum_{c \subset \bar{F}} A\left(T_{C}\right) .
$$

From Lemma IV.4, $L(D(t, G, T))=\sum_{c \subset \bar{p}} L_{C}(t)$. Integrating and using Lebesgue's convergence theorem

$$
\int_{0}^{\infty} L(D(t, G, T)) d t=\sum_{c \subset \bar{F}} \int_{0}^{\infty} L_{C}(t) d t=\sum_{C \subset \bar{F}} \int_{0}^{\infty} L\left(D\left(t-s_{C}, G, T_{C}\right) d\left(t-s_{C}\right) .\right.
$$

Since $C \subset \bar{F}$ the mappings $T_{C}$ satisfy the conditions of Theorem III hence

$$
\int_{0}^{\infty} L(D(t, G, T)) d t=\sum_{C \subset \bar{F}} A\left(T_{C}\right) .
$$

If $C$ is a proper cyclic element of $M$ and $b \in C, G(m(p), b, T)=s_{C}$ $+G\left(r_{C} \circ m(p), b, T_{C}\right)$, hence by Theorem II $F \cap C$ is either empty, the single point $r_{C} \circ m(p)$, or dense in $C$. Since $F$ is connected this implies that $\vec{F}$ is either a single point or an $A$-set in the terminology of [9]. By [9] there is a monotone retraction $r_{F}: M \rightarrow \bar{F}$ retracting $M$ onto $\bar{F}$. Let $T_{F}=1 \circ r_{F} \circ m$. Then $r_{F} \circ m$ is the monotone factor, 1 the light factor and $\bar{F}$ the middle space in a monotone light factorization of $T_{\boldsymbol{r}}$.

THEOREM V. Let $T$ be as in Theorem IV and suppose the hypothesis of Theorem IV holds. Let $F$ and $T_{F}$ be as described above. Then

$$
A\left(T_{F}\right)=\int_{0}^{\infty} L\left(D\left(t, G, T_{F}\right)\right) d t=\int_{0}^{\infty} L(D(t, G, T)) d t .
$$

If $\bar{F}$ is not a single point, $\bar{F}$ is a $A$-set and each boundary point of $\bar{F}$ separates $M$. Since $m(p) \in F$ and $r_{F}$ is a retraction $r_{F} \circ m(p)=m(p)$. These two statements imply that if $b \in \bar{F}$

$$
G\left(r_{F} \circ m(p), b, T_{F}\right)=G(m(p), b, T) .
$$

Hence $\left\{b \in \bar{F}: G\left(r_{F} \circ m(p), b, T_{F}\right)<\infty\right\}=F$.

Since $r_{C} \circ r_{F}=r_{C}$ if $C \subset \bar{F}$, by applying Theorem IV to the mapping $T_{P}$ it is seen that

$$
\int_{0}^{\infty} L\left(D\left(t, G, T_{F}\right)\right) d t=\sum_{C \subset \bar{F}} A\left(T_{C}\right) .
$$

Hence by the cyclic additivity theorem of $[9]$ it is seen that $\int_{0}^{\infty} L\left(D\left(t, G, T_{F}\right)\right) d t$ $=A\left(T_{F}\right)$. 


\section{REFERENCES}

1. J. Cecconi, La disuguaglianza di Cavalieri per la k-area secondo Lebesgue in un n-spazio, Ann. Mat. Pura Appl., Ser. 4 vol. 42 (1956) p. 189.

2. L. Cesari, Surface area, Annals of Mathematics Studies, Princeton University Press, 1956.

3. - Contours of a Frechet surface, Riv. Mat. Univ. Parma vol. 4 (1953) p. 173.

4. H. Federer, Surface area I, II, Trans. Amer. Math. Soc. vol. 55 (1944) p. 420.

5. - Measure and area, Bull. Amer. Math. Soc. vol. 58 (1952) p. 306.

6. —, On Lebesgue area, Ann. of Math. vol. 61 (1955) p. 289.

7. - Curvature measures, Trans. Amer. Math. Soc. vol. 93 (1959) p. 418.

8. M. Frechet, Sur quelques points du calcul fonctionnel, Rend. Circ. Mat. Palermo vol. 22 (1906) p. 1.

9. T. Rad6, Length and area, Amer. Math. Soc. Colloquium Publications, vol. 30, 1948.

10. E. Silverman, $A n$ intrinsic property for Lebesgue area, Riv. Mat. Univ. Parma vol. 2 (1951) p. 193.

11. - An intrinsic inequality for Lebesgue area, Pacific J. Math. vol. 6 (1956) 363.

12. S. Saks, The theory of the integral, Warsaw, 1937.

13. L. C. Young, Partial area. I, to appear in Riv. Mat. Univ. Parma.

14. H. Whitney, On totally differentiable smooth functions, Pacific J. Math. vol. 1 (1951) p. 143.

BROWN UNIVERSITY, Providence, Rhode IsLANd 